\title{
Construction of Barrier in a Fishing Game with Point Capture
}

\author{
Wenzhong Zha, Student Member, IEEE, Jie Chen, Senior Member, IEEE, Zhihong Peng Member, IEEE,, \\ and Dongbing Gu, Senior Member, IEEE
}

\begin{abstract}
This paper addresses a particular pursuit-evasion game, called as "fishing game" where a faster evader attempts to pass the gap between two pursuers. We are concerned with the conditions under which the evader or pursuers can win the game. This is a game of kind in which an essential aspect, barrier, separates the state space into disjoint parts associated with each player's winning region. We present a method of explicit policy to construct the barrier. This method divides the fishing game into two sub-games related to the included angle and the relative distances between the evader and the pursuers, respectively, and then analyzes the possibility of capture or escape for each sub-game to ascertain the analytical forms of the barrier. Furthermore, we fuse the games of kind and degree by solving the optimal control strategies in the minimum time for each player when the initial state lies in their winning regions. Along with the optimal strategies, the trajectories of the players are delineated and the upper bounds of their winning times are also derived.
\end{abstract}

Index Terms-Fishing game, pursuit-evasion games, barrier, point capture, winning regions, optimal strategies.

\section{INTRODUCTION}

$\mathbf{R}$ ESEARCHES in the areas of robotics and control are usually related to controlling multiple mobile agents to complete some tasks in conflicting scenarios. For example, in RoboCup two robot soccers try to get the ball from an opponent, in policing two autonomous vehicles attempt to trace a crime target, in military confrontation two missiles aim to intercept an enemy target, and from the view of escape, in collision avoidance a robot intends to pass through two moving obstacles, etc. These application scenarios can be generalized to a kind of pursuit-evasion game, where one evader must pass the gap between two pursuers.

Manuscript received September 6, 2015; revised February 4, 2016; accepted March 21, 2016. This work was supported by British Council Sino-UK Higher Education Research Partnership for PhD Studies, China Scholarship Council, Foundation for Innovative Research Groups of the National Natural Science Foundation of China (Grant No.61321002), Projects of Major International (Regional) Joint Research Program NSFC (Grant No.61120106010), Beijing Education Committee Cooperation Building Foundation Project, Program for Changiiang Scholars and Innovative Research Team in University under Grant IRT1208, Chang Jiang Scholars Program, Beijing Outstanding Ph.D. Program Mentor Grant (Grant No.20131000704) and National Natural Science Foundation of China (Grant No.61203078).

W. Zha, J. Chen and Z. Peng are with School of Automation, Beijing Institute of Technology, Beijing 100081, China, and also with the Key Laboratory of Intelligent Control and Decision of Complex Systems, Beijing 100081, China (e-mail: zhawenzhong@126.com; chenjie@bit.edu.cn; peng@bit.edu.cn).

D. Gu is with School of Computer Science and Electronic Engineering, University of Essex, Wivenhoe Park, Colchester, CO4 3SQ, UK (e-mail: dgu@essex.ac.uk).

Color versions of one or more of the figures in this paper are available online at http://ieeexplore.ieee.org.
This paper considers such a particular pursuit-evasion game with point capture. Specifically, the three players move in the plane with constant speeds and unlimited directions. When the minimum distance between the evader and the pursuers is maintained not equal to zero and the evader travels through the gap between the two pursuers (i.e., the included angle between them increases from $<\pi$ to $>\pi$ ), the evader wins; otherwise, the pursuers win. This game is analogous to a natural scene of two fishers using a fishing net to catch one fish, where the fish endeavours to escape from a gap between the fishers. Thus, it can be vividly called as "fishing game" in this paper.

Different from general pursuit-evasion games, there are two competition focuses for the players in fishing game: one is the relative distances which the pursuers try to minimize while the evader attempts to maximize; the other one is the gap between the two pursuers which the evader seeks to pass through while the pursuers strive to narrow. In fact, the pursuit-evasion game with more than two pursuers can be regarded as a combination of multiple fishing games. Therefore, the above adversarial interactions among three players are available for representing some challenges encountered in control problems, such as cooperative besieging and capturing of multi-robot $[1,2]$, paths coordination of multi-agents [3, 4], and collision avoidance of moving vehicles in hazardous circumstances $[5,6]$. Naturally, in a fishing game if the pursuers are faster than the evader, they will always win from any given initial position of the players. But a close situation that the pursuers possess superiority in numbers while the evader also has an advantage in speed, is more attractive to us because we cannot immediately estimate the possibility of capture or escape for the players. This is a typical game of kind and is the focus of this paper.

It is well-known that in Isaacs's book [7] the core concept of the game of kind, barrier, separates the state space into two parts: capture zone and escape zone. If the initial state lies in the capture zone, then there exists a suitable strategy for the pursuer such that capturing the evader can be guaranteed no matter what strategy the evader will take. Similarly, if the initial state lies in the escape zone, then the evader also has a suitable strategy to carry out such that its escape can be guaranteed regardless of the pursuer's strategy. Consequently, when the initial state is on the barrier, the intersection of their winning regions, the pursuer must adopt an optimal strategy to prevent the state entering the escape zone; simultaneously, the evader should also exert itself to keep the state out of the capture zone. The nature reflected by the players competing on the barrier is called as "semipermeability", and the barrier is precisely a semipermeable surface. 
The primary goal of this paper is constructing such a barrier to delineate the winning region for each player in fishing game. The classic Isaacs's approach [7] to construct barrier is starting from a point of the boundary of the usable part (BUP) of target set (or terminal manifold) and then integrating the so-called retrogressive path equations (RPEs). However, it is difficult to solve the RPEs analytically in fishing game because the target set involves not only the relative distances but also the included angle between the pursuers and the evader. For this reason, we present a direct and effective method, namely method of explicit policy, demonstrates the possibility of capture or escape by exhibiting some particular strategies or policies of the players. Specifically, we divide the game into two subgames which are related to the relative distances and the included angle, respectively. For each sub-game, we analyze the possibility of capture or escape and obtain the analytical forms of winning regions for the players, and then we ascertain a precise description of the barrier by synthesizing these winning regions. The above results comprise an open-loop solution in Nash equilibrium [8].

Additionally, another contribution of this paper is the fusion of the games of kind and degree in fishing game, where each player seeks to achieve success in the minimum time when the initial state lies in their winning regions. We partition the state space into four zones and then attain the optimal trajectories associated with the optimal control strategies of the players for every zone, which are the closed-loop solutions of fishing game. To be the best of our knowledge, this paper is the first that provides such a complete solution (including the openloop solution and the closed-loop solution) to the fishing game, where the results obtained can be useful for giving insight into general problems of cooperative besieging and capturing for multi-robot systems.

This paper is organized as follows. In Section II, related work is reviewed. In Section III, the fishing game in question is defined and its formulation is discussed. In Section IV, the analytical forms of the winning regions and the barrier are obtained by analyzing the control policies of the players for each sub-game. In Section V, the closed-loop solutions, i.e., the optimal strategy and the trajectory of each player with respect to the minimum time are solved. Finally, in Section VI, conclusions and future work are summarized.

\section{RELATED WORK}

In terms of the game of kind, Isaacs [7] introduces several methods to construct the barriers including natural barriers, artificial barriers and envelope barriers. He provides a plethora of examples to illustrate the semipermeability of barriers, such as the game of homicidal chauffeur, the game of two cars, the lifeline game, the deadline game, etc. Since the pioneering work, there have been a number of efforts to characterize the barriers in pursuit-evasion games. For example, a complete solution in the game of homicidal chauffeur is obtained by Merz [9], and all the types of singular curves appearing in the game are analyzed, involving the dispersal, universal, equivocal, switch and focal curves. Families of semipermeable curves in the game are deliberated by Patsko and Turova [10], which are useful for game problems with various performance indices and the determination of capture sets. The surveillanceevasion problem with the same dynamics is considered by Lewin and Breakwell [11] and other variants of the game are investigated by David [12], Parsko and Turova [13], and Exarchos et al. [14]. For the deadline game, Breakwell [15] analyzes the optimal trajectories and barriers for a series of games posed by Isaacs (e.g. the one-sided deadline game, the cornered rat game, the game of patrolling a channel), then he extends the results to a game of kind with two pursuers and one evader in [16]. Moreover, constructions of barriers in the game of two cars and the lifeline game can be consulted in [17-21] and [22-24], respectively.

In addition, some special games related to practical applications are also handled by using Isaacs's approach. For example, Bhattacharya, Hutchinson and Başar [25-27] concern the visibility based pursuit-evasion game in an environment containing obstacles and present the necessary and sufficient conditions for surveillance and escape. Based on these findings, they construct a value function of the game and obtain optimal trajectories for the players as well as the dispersal surfaces arising in the solutions [28-29]. Shinar et al. [30-34] analyze many pursuit-evasion games of kind with different dynamics of missiles and aircrafts, in particular, a hybrid evader who has two possible dynamics considered in [34] is a very interesting viewpoint.

The aforementioned methods to construct barriers are often starting from a point of the BUP of target set and then integrating the RPEs, however, become more and more difficult to take effect with the increasing complexity of pursuit-evasion problems. The main reasons are that: the RPEs, derived from the Hamilton-Jacobi-Isaacs (HJI) equations, are usually hard to solve analytically for somewhat sophisticated problems, especially in multi-agent systems. Since the solving difficulty depends on the dynamics and the number of players, the multi-player games of kind may also suffer from the curse of dimensionality. To date, a general solution to multi-player pursuit-evasion games is not yet available.

Generally, there are three ways to cope with the above problems. The first one is dividing the game into several subgames and then analyzing the optimal behaviors of the players for each sub-game. For example, Hagedorn and Breakwell [16] undertake Isaacs's inference [7] for a game of kind with two pursuers and one evader (similar to fishing game) that the optimal trajectories of the players have two phases: first, straight-line phase during which the distances between the pursuers and the evader are decreasing, and second, curved phase during which one of these relative distances maintains equal to the capture radius. That is, the target in the game is divided into two successive sub-targets. Bhattacharya [35] also uses Isaacs's technique to address the problem of connectivity maintenance game with two aerial vehicles and one jammer, where the target set of the game is represented as the union of two smooth termination surfaces. In $[36,37]$, Tomlin's group presents an open-loop formulation for a reach-avoid game as a pair of games which are conservative for the attacking and defending players respectively. Then they treat the capturethe-flag game as a multi-stage reach-avoid game in [38] and 
develop a complete solution of the game in [1].

The second way is using the method of explicit policy to avoid solving HJI equations. For instance, Turetsky [39] obtains the necessary and sufficient conditions for having a nontrivial capture zone by constructing a class of time-varying feedback pursuit strategies. Huang et al. [40, 41] present a decentralized control scheme for multiple pursuers based on Voronoi partition of the game domain, a bounded convex polytope, to guarantee capturing a single evader. Furthermore, they consider the cooperation among the pursuers [42] in a bounded, simply-connected planar domain, and obtain the conditions of guaranteeing capture. Alexander, Bishop and Ghrist [43] use convex-geometric techniques to provide a necessary and sufficient condition for the eventual capture in multi-pursuer one evader games on convex Euclidian domains of arbitrary dimension and shape. Kim and Sugie [44] propose a cyclic pursuit strategy for multiple pursuers to capture a single target in 3D space, which only uses the local information to generate the global group behaviour. Bopardikar et al. [45] address a cooperative Homicidal Chauffeur game with multiple pursuers and one evader. Inspired by hunting and foraging behaviours of fish species, they present a multiphase cooperative strategy to confine the evader to a bounded region. In [46], they study the pursuit-evasion problems in discrete time and continuous space, where at least $k$ pursuers are required to reach the evader's location simultaneously, and provide a sufficient condition for this kind of $k$-capture. Analogously, Özgüler and Yıldız [47] also investigate the foraging swarm behaviour in scope of noncooperative game, and obtain the Nash equilibrium solution by explicitly describing a class of strategies. The method of explicit policy generally, involving geometric analysis, is computationally efficient in generating control strategies, while are limited to relatively simple game environment and simple motion models of the players.

The last approach is to solve the numerical approximation of HJI equations. Based on the theory of viscosity solution for HJI equations [48], Bernhard, Crepey and Rapaport [49] present a numerical method for the computation of the value function using Kruzkov transformation, and determine the barrier of this game in time by depicting the zero level set of the value function. Bhattacharya, Başar and Falcone [50] address a vision-based target tracking problem, and use a fully discrete semi-Lagrangian scheme to compute the Kruzkov transformation of the value function numerically. Kumkov, Ménec and Patsko [51] implement a procedure of dynamic programming to construct level sets of the value function numerically for a class of linear differential games with fixed termination instant. Zhang, Cui and Luo [52] propose a near-optimal control scheme, utilizing the single-network adaptive dynamic programming (ADP), to solve the nonzero-sum differential games of continuous-time nonlinear systems. For a nonzerosum game with unknown dynamics, Zhao et al. [53] present a single-network ADP with experience replay algorithm to solve the coupled nonlinear HJI equations. Mitchell, Bayen and Tomlin [54] describe and implement an algorithm to compute a so-called reachable set which is the zero sublevel set of the viscosity solution of a time-dependent HJI partial differential equation. In [55] they adopt this reachability algorithm to index one differential algebraic equation, and approximate the backward reachable set on a constraint manifold with lower dimension and in the full dimensional state space, respectively. Other approximation methods such as neural network [56], Q-learning [57] and reinforcement learning [58, 59] are also being concerned in recent years.

In fact, the fishing game is related to the classic one-sided deadline game described by Isaacs [7], or more closely to a pursuit game proposed by Breakwell [16]. But differing from their assumption of some positive capture radius, point capture, i.e., the capture radius is zero, is considered in our fishing game. Thus, it is more challenging to capture a faster evader for the pursuers. Moreover, their handling method of dividing the target into two successive sub-targets is also not suitable for fishing game.

Generally, analysis for a game of kind highlights the tradeoffs between problem characteristics, probability for capture or escape, solutions optimality, and computational complexity. Comparing the various methods presented in the aforementioned references, we find that the methods associated with solving HJI equations, whether analytically or numerically, are complicated to obtain a complete solution for the fishing game. Instead, the method of explicit policy can effectively avoid such a dilemma, even though some properties may be somewhat tortuous to prove geometrically. The following sections discuss the concrete implementation of this method, which shows that both the form of solution and the representation of barrier are quite concise.

\section{PROBLEM Formulation}

In this section, we present the problem formulation of fishing game. Consider two identical pursuers $P_{1}, P_{2}$ and one evader $E$ moving in the plane (Fig. 1), the evader has speed $v_{e}$ and both pursuers have speed $v_{p}$. Their control variables are the directions of their velocity vectors respectively. The evader attempts to pass the gap between the two pursuers, i.e., crossing the line segment $P_{1} P_{2}$ from left to right as shown in Fig. 1 (segment $P_{1} P_{2}$ is time-varying), whilst avoiding being captured by the pursuers. On the contrary, the pursuers strive to capture the evader whilst preventing the evader from threading.

The kinematic equations of the pursuers and the evader in the realistic game space are given by:

$$
\begin{array}{ll}
\dot{x}_{1}=v_{p} \sin \phi_{1}, & \dot{y}_{1}=-v_{p} \cos \phi_{1} \\
\dot{x}_{2}=v_{p} \sin \phi_{2}, & \dot{y}_{2}=-v_{p} \cos \phi_{2} \\
\dot{x}_{e}=v_{e} \sin \varphi, & \dot{y}_{e}=-v_{e} \cos \varphi
\end{array}
$$

where the positions of the players are denoted as $P_{1}\left(x_{1}, y_{1}\right)$, $P_{2}\left(x_{2}, y_{2}\right)$ and $E\left(x_{e}, y_{e}\right)$, and the corresponding control variables are denoted as $\phi_{1}, \phi_{2}$ and $\varphi$, respectively.

The problems in fishing game are that:

1) What conditions of the initial state can guarantee the win of the pursuers or the evader when they play optimally?

2) If the win can be assured, what strategies should be adopted by the pursuers or the evader to achieve success in the minimum time. 


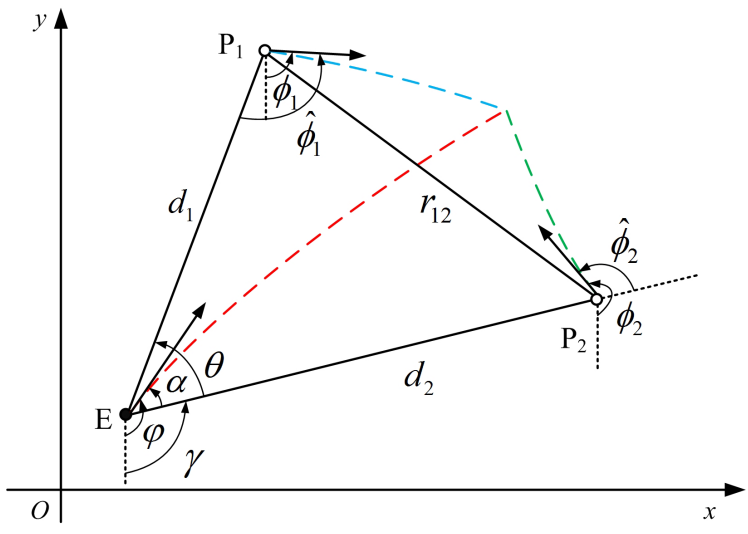

Fig. 1. Fishing game in the fixed reference system, where $\varphi, \phi_{1}$ and $\phi_{2}$ are the moving directions of players $E, P_{1}$ and $P_{2}$ in the realistic game space, respectively. $\alpha, \hat{\phi}_{1}$ and $\hat{\phi}_{2}$ are the alternative control variables of the players, defined as the relative headings of $E, P_{1}$ and $P_{2}$ from the vectors $\overrightarrow{E P_{2}}, \overrightarrow{P_{1} E}$ and $\overrightarrow{E P_{2}}$, respectively. $\theta$ denotes the included angle $\angle P_{2} E P_{1}$, and $\gamma$ denotes the angle of vector $\overrightarrow{E P_{2}} . d_{1}, d_{2}$ and $r_{12}$ are the lengths of segments $E P_{1}$, $E P_{2}$ and $P_{1} P_{2}$, respectively.

Since the pursuers' win can always be attained from any given initial state for $v_{p}>v_{e}$, we only concern the case $v_{p} \leq$ $v_{e}$ and use $a$ to denote the ratio of $v_{p}$ to $v_{e}, a=v_{p} / v_{e} \leq 1$. The winning strategy of the pursuers for $a>1$ is to move towards the evader individually.

To represent the dynamics of fishing game more compactly, a reduced state space can be formed by the relative distances $d_{1}, d_{2}$ and the included angle $\theta$ (see Fig. 1) in which the dynamics are derived as follows:

$$
\begin{array}{rlrl}
\dot{d}_{1}= & -v_{p} \cos \hat{\phi}_{1}-v_{e} \cos (\theta-\alpha), & d_{1}\left(t_{0}\right)=d_{1}^{0} \\
\dot{d}_{2}=v_{p} \cos \hat{\phi}_{2}-v_{e} \cos \alpha, & d_{2}\left(t_{0}\right)=d_{2}^{0} \\
\dot{\theta}=-\frac{v_{p}}{d_{1}} \sin \hat{\phi}_{1}+\frac{v_{e}}{d_{1}} \sin (\theta-\alpha)- & \\
& -\frac{v_{p}}{d_{2}} \sin \hat{\phi}_{2}+\frac{v_{e}}{d_{2}} \sin \alpha, & \theta\left(t_{0}\right)=\theta_{0}
\end{array}
$$

where $\alpha, \hat{\phi}_{1}$ and $\hat{\phi}_{2}$ are the alternative control variables of the players, defined as the relative headings of $E, P_{1}$ and $P_{2}$ from the vectors $\overrightarrow{E P_{2}}, \overrightarrow{P_{1} E}$ and $\overrightarrow{E P_{2}}$, respectively. As shown in Fig. $1, \alpha=\varphi-\gamma, \hat{\phi}_{1}=\phi_{1}+\pi-\theta-\gamma$ and $\hat{\phi}_{2}=\phi_{2}-\gamma(\gamma$ is the angle of vector $\left.\overrightarrow{E P_{2}}\right) . \theta$ is the included angle between the vectors $\overrightarrow{E P_{1}}$ and $\overrightarrow{E P_{2}}, \theta=\operatorname{Arg}\left(\overrightarrow{E P_{1}}\right)-\operatorname{Arg}\left(\overrightarrow{E P_{2}}\right) \cdot \operatorname{Arg}(\cdot)$ is the principal value of the argument of a vector, within the range $(-\pi, \pi]$. The full state space is denoted by $\Omega=\left\{d_{1} \geq\right.$ $\left.0, d_{2} \geq 0,-\pi<\theta \leq \pi\right\} \in \mathbb{R}^{3}$.

Without loss of generality, we assume that the initial included angle $\theta_{0} \in[0, \pi)$. As for the case of $(-\pi, 0)$, it is easy to obtain a similar result according to symmetry. Thus, from the beginning of the fishing game with point capture, $\min \left\{d_{1}^{0}, d_{2}^{0}\right\}>0$ and $0 \leq \theta_{0}<\pi$, if

a) $\min \left\{d_{1}, d_{2}\right\}>0$ and $\theta=\pi$, the evader wins;

b) else if $\min \left\{d_{1}, d_{2}\right\}=0$, or the state maintains $\left\{d_{1}>\right.$ $0, d_{2}>0$ and $\left.\theta<\pi\right\}$ indefinitely, the pursuers win.

The above are regarded as the termination conditions of fishing game.

\section{WINNING REGIONS AND BARRIER}

The problem formulated in the previous section is a game of kind, where the entire state space is split by a barrier into two disjoint regions: the capture zone and the escape zone. In this work, our primary goal is to construct such a barrier, and subsequently solve the optimal feedback strategies for the pursuers and the evader when the initial state is in their winning regions, respectively.

In a general game of kind, target set refers to a set of the states satisfying a termination condition of the game. The pursuer wishes to steer the state to the target set, while the evader hopes to keep it out of that set. The boundary of target set, i.e., terminal manifold, is composed of two parts: one is called as usable part (UP), on which the pursuer can force the state to penetrate into the interior of target set despite the evader's strategy; the other one is called as nonuseable part (NUP), on which the evader can frustrate the penetration regardless of the pursuer's strategy. Thus, each player exerts its optimal endeavor to make the state moving tangentially to the boundary of target set, where the points of tangency constitute the boundary of usable part (BUP). Obviously, the BUP of target set is semipermeable and can be used as the initial curve for the barrier within the reversed time. Starting from a point of the BUP, the barrier can be constructed by integrating the retrogressive path equations (RPEs).

That is the classic Isaacs's approach [7] to construct the barrier, nonetheless, is unsuited to fishing game directly, because the corresponding RPEs associated with the reduced state variables $\left(d_{1}, d_{2}\right.$ and $\left.\theta\right)$ are hard to solve analytically. Instead, we can divide the game into two sub-games from the perspective of the pursuers: the first one involves the competition on the included angle $\theta$ that the pursuers attempt to decrease it while the evader hopes to increase it; The second one is focused on the distance $\min \left\{d_{1}, d_{2}\right\}$ that the pursuers try to make it zero, while the evader strives to keep it positive and increase the angle $\theta$. Since the two subgames are not successive, there will be some intersections between their solutions. Thus, our method is solving these two sub-games of kind, respectively, and then integrating the corresponding solutions (including the optimal strategies, the winning conditions) into a complete solution of the whole game.

\section{A. Competition on the Included Angle $\theta$}

In the first sub-game of kind, the termination conditions can be reduced to a discrimination of the included angle $\theta$ : when $\theta<\pi$ always holds, the pursuers win; when $\theta=\pi$, the evader wins. Now, we begin to solve the fishing game based on the changes of $\theta$, and derive the main equation:

$$
\begin{aligned}
\min _{\hat{\phi}_{1}, \hat{\phi}_{2}} \max _{\alpha}[ & -\frac{v_{p}}{d_{1}} \sin \hat{\phi}_{1}-\frac{v_{p}}{d_{2}} \sin \hat{\phi}_{2}+ \\
& \left.+\frac{v_{e}}{d_{1}} \sin (\theta-\alpha)+\frac{v_{e}}{d_{2}} \sin \alpha\right]=0
\end{aligned}
$$

so that the optimal control strategies of the pursuers and the evader are

$$
\hat{\phi}_{1}^{*}=\frac{\pi}{2}, \quad \hat{\phi}_{2}^{*}=\frac{\pi}{2}
$$




$$
\sin \alpha^{*}=\frac{d_{1}-d_{2} \cos \theta}{r_{12}}, \quad \cos \alpha^{*}=\frac{d_{2} \sin \theta}{r_{12}}
$$

where $r_{12}=\sqrt{d_{1}^{2}+d_{2}^{2}-2 d_{1} d_{2} \cos \theta}$ is the distance between the pursuers $P_{1}$ and $P_{2}$, and symmetrically

$$
\sin \left(\theta-\alpha^{*}\right)=\frac{d_{2}-d_{1} \cos \theta}{r_{12}}, \cos \left(\theta-\alpha^{*}\right)=\frac{d_{1} \sin \theta}{r_{12}} .
$$

Thus the main equation (7) can be rewritten as

$$
-\frac{v_{p}}{d_{1}}-\frac{v_{p}}{d_{2}}+\frac{v_{e} r_{12}}{d_{1} d_{2}}=0
$$

then,

$$
r_{12}=a\left(d_{1}+d_{2}\right)
$$

where $a=v_{p} / v_{e}$ is the speed ratio of the pursuers to the evader. Consider the expression of $r_{12}$, from (12) we have

$$
\cos \theta=\cos \Theta=\frac{\left(1-a^{2}\right)\left(d_{1}+d_{2}\right)^{2}}{2 d_{1} d_{2}}-1 .
$$

Here, we call $\Theta$ the non-escape angle according to the following theorem:

Theorem 4.1: If the initial state satisfies

$$
r_{12}\left(t_{0}\right) \leq a\left(d_{1}\left(t_{0}\right)+d_{2}\left(t_{0}\right)\right)
$$

or

$$
\theta\left(t_{0}\right) \leq \Theta\left(t_{0}\right)
$$

then the pursuers' win can be guaranteed as long as they adopt the strategy (8).

Proof: As condition (14) is equivalent to condition (15), we only prove the latter. Firstly, if $\theta\left(t_{0}\right)<\Theta\left(t_{0}\right)$, from the formula (13) and the main equation (11), we have

$$
\dot{\theta}\left(t_{0}\right)=-\frac{v_{p}}{d_{1}}-\frac{v_{p}}{d_{2}}+\frac{v_{e} r_{12}}{d_{1} d_{2}}<0 .
$$

Substituting the optimal control strategies (8) and (9) into the differential equations (4) and (5), then

$$
\dot{d}_{1}=-\frac{v_{e} d_{1}}{r_{12}} \sin \theta, \quad \dot{d}_{2}=-\frac{v_{e} d_{2}}{r_{12}} \sin \theta
$$

and $\dot{d}_{1} / \dot{d}_{2}=d_{1} / d_{2}$. Let $b=d_{1} / d_{2}$, since the game continues in terms of $d_{1}>0, d_{2}>0$ and $0<\theta<\pi$, we have

$$
\dot{b}=\frac{\dot{d}_{1} d_{2}-\dot{d}_{2} d_{1}}{d_{2}^{2}}=0 .
$$

Besides, the formula (13) can be transformed to

$$
\cos \Theta=\frac{\left(1-a^{2}\right)(1+b)^{2}}{2 b}-1 .
$$

Therefore, the value of $\Theta(t)$ will be an invariant when the pursuers and the evader play optimally. And obviously $\cdots<$ $\theta\left(t_{2}\right)<\theta\left(t_{1}\right)<\theta\left(t_{0}\right)<\Theta\left(t_{0}\right)=\Theta\left(t_{1}\right)=\Theta\left(t_{2}\right)=\cdots$, i.e., the value of $\theta(t)$ will decrease monotonically with time. Further, based on the concept of Nash equilibrium, we are sure that the objective of $\theta\left(t_{f}\right)<\pi$ can be achieved by the pursuers no matter what strategy of the evader.

Secondly, if $\theta\left(t_{0}\right)=\Theta\left(t_{0}\right)$, then $\dot{\theta}\left(t_{0}\right)=0$ when all the players adopt the optimal strategies. It means that the value of $\theta(t)$ will remain unchanged. But $\dot{d}_{1}<0$ and $\dot{d}_{2}<0$, so the pursuers can still make $\min \left\{d_{1}, d_{2}\right\}=0$ to terminate the game in finite time.
Remark 4.1: According to the formula (16), for a meaningful $\cos \Theta \leq 1, a$ and $b$ must satisfy

$$
\frac{1-a}{1+a} \leq b \leq \frac{1+a}{1-a}, \quad(a<1) .
$$

If the above inequalities are not satisfied, for example,

$$
b>\frac{1+a}{1-a}>1, \text { i.e., } a<\frac{b-1}{b+1}
$$

then

$$
\begin{aligned}
\left.\dot{\theta}\right|_{\hat{\phi}_{1}^{*}, \hat{\phi}_{2}^{*}, \alpha^{*}} & =-\frac{v_{p}}{d_{1}}-\frac{v_{p}}{d_{2}}+\frac{v_{e} r_{12}}{d_{1} d_{2}} \\
& =\frac{v_{e}}{d_{1}}\left[\sqrt{b^{2}+1-2 b \cos \theta}-a(1+b)\right] \\
& >\frac{v_{e}}{d_{1}}\left[\sqrt{b^{2}+1-2 b \cos \theta}-(b-1)\right] \\
& \geq 0
\end{aligned}
$$

that is, $\dot{\theta}>0$ will occur regardless of the value of $\theta$. This implies that the condition (14) or (15) has entailed the constraints of $b$ in (17). Moreover, it also shows that if the pursuers hope to win they should make $b$, the ratio of $d_{1}$ to $d_{2}$, close to 1 when $a$ is small.

Remark 4.2: From the formula (13), given $b$, the value of the non-escape angle $\Theta$ increases monotonically with $a$, and when $a=1, \Theta=\pi$; Given $a, \Theta$ will achieve the maximum value $\arccos \left(1-2 a^{2}\right)$ when $b=1$.

Remark 4.3: Note that when $\theta=0$, if $r_{12} \leq a\left(d_{1}+d_{2}\right)$ the pursuers can still win the game since thereafter $\theta \leq 0$. The three players' trajectories associated with the optimal strategies (8) and (9) are sketched in Fig. 2. When $r_{12}<a\left(d_{1}+d_{2}\right)$ (see Fig. 2(a)), $\dot{d}_{1}>0, \dot{d}_{2}>0$ and $\dot{\theta}<0$, thus the evader will not able to go through $P_{1}$ and $P_{2}$ whether on the left or the right. In particular, when $r_{12}=a\left(d_{1}+d_{2}\right)$ (see Fig. 2(b)), their trajectories will be circles with the center at the midpoint of segment $P_{1} P_{2}$. The evader still can not win the game. Actually, in the next section we will see that the pursuers can make $r_{12}=0$ to terminate the game as long as they go straight towards each other.

From Theorem 4.1, the winning region of the pursuers denoted by $\mathscr{D}_{p}^{1}$ can be described as follows

$$
\begin{aligned}
\mathscr{D}_{p}^{1} & =\left\{d_{1}, d_{2}, \theta \mid \sqrt{d_{1}^{2}+d_{2}^{2}-2 d_{1} d_{2} \cos \theta} \leq a\left(d_{1}+d_{2}\right)\right\} \\
& =\left\{d_{1}, d_{2}, \theta \mid \cos \theta \geq \frac{\left(1-a^{2}\right)\left(d_{1}+d_{2}\right)^{2}}{2 d_{1} d_{2}}-1\right\}
\end{aligned}
$$

and its boundary is denoted by $\mathscr{B}_{1}$,

$$
\mathscr{B}_{1}=\left\{d_{1}, d_{2}, \theta \mid \cos \theta=\frac{\left(1-a^{2}\right)\left(d_{1}+d_{2}\right)^{2}}{2 d_{1} d_{2}}-1\right\} .
$$

Obviously, if $a=1, \mathscr{D}_{p}^{1}$ will be the entire state space subtracting the evader's winning condition $\left\{d_{1}>0, d_{2}>\right.$ 0 and $\theta=\pi\}$. So, we will suppose $a<1$ in the rest of this paper.

Next, we analyze the complementary region of $\mathscr{D}_{p}^{1}$. At this time, even though $\dot{\theta}>0$ when the players adopt strategies (8) and (9), we worry about that the evader still fails in $\theta=\pi$ because $\min \left\{d_{1}, d_{2}\right\}$ may be equal to zero previously. But since the maximum non-escape angle, $\max \Theta$, is equal to 


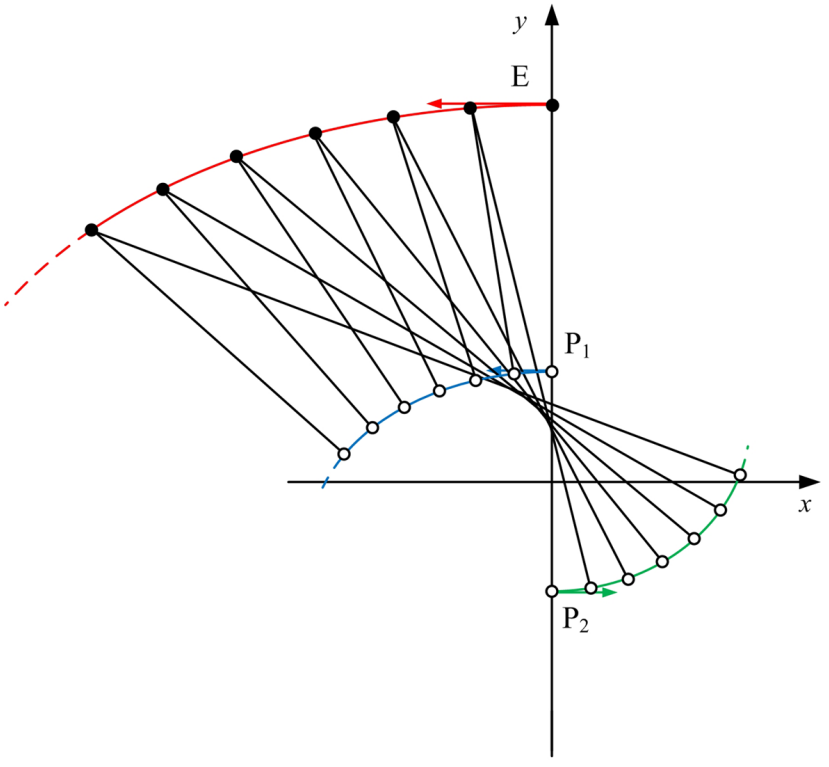

(a) $r_{12}<a\left(d_{1}+d_{2}\right)$

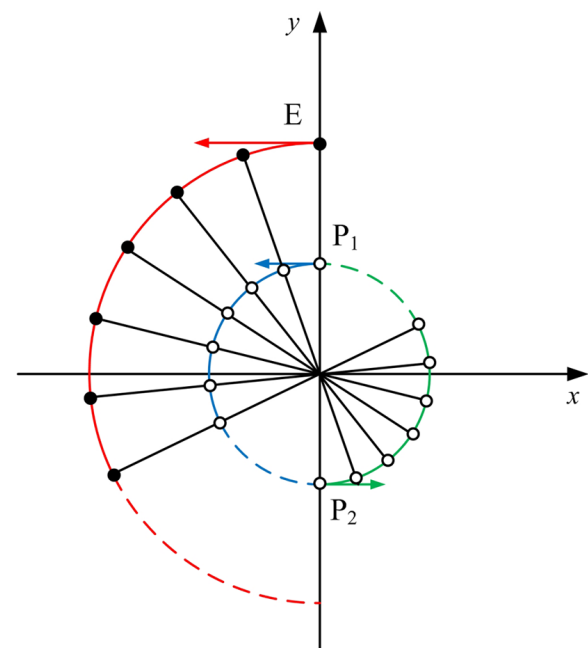

(b) $r_{12}=a\left(d_{1}+d_{2}\right)$

Fig. 2. The trajectories of the players using strategies (8) and (9) after $\theta=0$ and $r_{12} \leq a\left(d_{1}+d_{2}\right)$, where the red curve with black spots represents the evader's trajectory, the blue curve and the green curve with circles represent the trajectories of the pursuers $P_{1}$ and $P_{2}$ respectively. When (a) $r_{12}<a\left(d_{1}+d_{2}\right)$, the evader moves away from the gap between $P_{1}$ and $P_{2}$ gradually. Symmetrically, this dilemma will also be encountered if it turns right initially. When (b) $r_{12}=a\left(d_{1}+d_{2}\right)$, the players $E, P_{1}$ and $P_{2}$ remain in a straight line, and their trajectories will be circles with the center at the midpoint of segment $P_{1} P_{2}$.

$\arccos \left(1-2 a^{2}\right)$ for any given $a<1$, the evader's win can be ensured according to the following theorem:

Theorem 4.2: If the initial state satisfies

$$
\theta\left(t_{0}\right)>\arccos \left(1-2 a^{2}\right)
$$

then there exists a suitable strategy for the evader to escape through the pursuers no matter what strategies the pursuers will take.

Proof: This theorem can be proved briefly by introducing a well-known concept of Apollonius circle [7], which is the locus of a point that the ratio of the distances between this point to two fixed points is constant. As shown in Fig. 3, the ratio is equal to $a<1$, the centers of circle $Q_{1}$ and circle $Q_{2}$ are

$$
\begin{aligned}
Q_{1} & =\left(\frac{x_{1}-a^{2} x_{e}}{1-a^{2}}, \frac{y_{1}-a^{2} y_{e}}{1-a^{2}}\right) \\
Q_{2} & =\left(\frac{x_{2}-a^{2} x_{e}}{1-a^{2}}, \frac{y_{2}-a^{2} y_{e}}{1-a^{2}}\right)
\end{aligned}
$$

and the corresponding radiuses are

$$
R_{1}=\frac{a d_{1}}{1-a^{2}} \quad R_{2}=\frac{a d_{2}}{1-a^{2}} .
$$

Further, it is easy to obtain that

$$
\sin \angle P_{1} E A=\sin \angle P_{2} E B=\sin \xi=a .
$$

Because $\theta>\arccos \left(1-2 a^{2}\right)$, we have $\theta>2 \xi$ and $\angle A E B>$ 0 . Thus, if the evader moves straight towards the interspace between $A$ and $B$, i.e., its heading satisfies $\xi<\alpha^{*}<\theta-\xi$, the pursuers will never catch up with it.

Similarly, from Theorem 4.2, the evader's winning region denoted by $\mathscr{D}_{e}^{1}$ is given by

$$
\mathscr{D}_{e}^{1}=\left\{d_{1}, d_{2}, \theta \mid d_{1}>0, d_{2}>0, \theta>\arccos \left(1-2 a^{2}\right)\right\}
$$

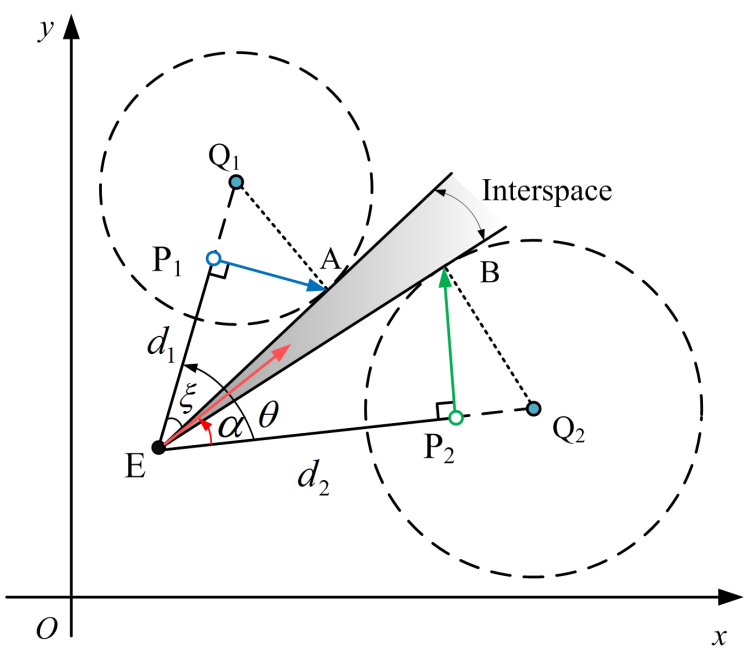

Fig. 3. The optimal strategy of the evader when the state satisfies $\theta>$ $\arccos \left(1-2 a^{2}\right)$, where the dashed circles with centers $Q_{1}$ and $Q_{2}$ are the Apollonius circles. $A$ and $B$ are the intersection points of the tangents of point $E$ to circle $Q_{1}$ and circle $Q_{2}$, respectively. The ratio of $\left|A P_{1}\right|$ (or $\left|B P_{2}\right|$ ) to $|A E|$ (or $|B E|$ ) is equal to $a$. If the evader moves along the direction of vector $\overrightarrow{E A}$, it will meet the pursuer $P_{1}$ at pint $A$. That is similar to the direction of $\overrightarrow{E B}$. Thus, the optimal strategy of the evader will be going towards the interspace between $A$ and $B$ (the shaded area), then the pursuers will never catch up with it.

whose boundary, denoted by $\mathscr{B}_{2}$, is

$$
\mathscr{B}_{2}=\left\{d_{1}, d_{2}, \theta \mid d_{1}>0, d_{2}>0, \theta=\arccos \left(1-2 a^{2}\right)\right\} .
$$

Remark 4.4: When the state lies in the region $\Omega \backslash\left\{\mathscr{D}_{p}^{1} \cup \mathscr{D}_{e}^{1}\right\}$ ( $\Omega$ is the full state space), i.e., $\Theta<\theta \leq \arccos \left(1-2 a^{2}\right)$, the possibility of capture or escape is uncertain in the first subgame, because the pursuers might capture the evader within the 
termination condition $\min \left\{d_{1}, d_{2}\right\}=0$, or the evader might pass between the pursuers successfully. However, an exception will arise in a particular assumption that the movement of the evader is confined to a straight line, then the pursuers' win can be assured by choosing some suitable strategies when the initial state is in $\Omega \backslash \mathscr{D}_{e}^{1}$, i.e., $\theta \leq \arccos \left(1-2 a^{2}\right)$. In this case, the first sub-game is equivalent to the whole game and the barrier of the game is $\mathscr{B}_{2}$. An extreme situation is shown in Fig. 4, where $\theta=\arccos \left(1-2 a^{2}\right)$ and there is no interspace between $A$ and $B$ for the evader to escape.

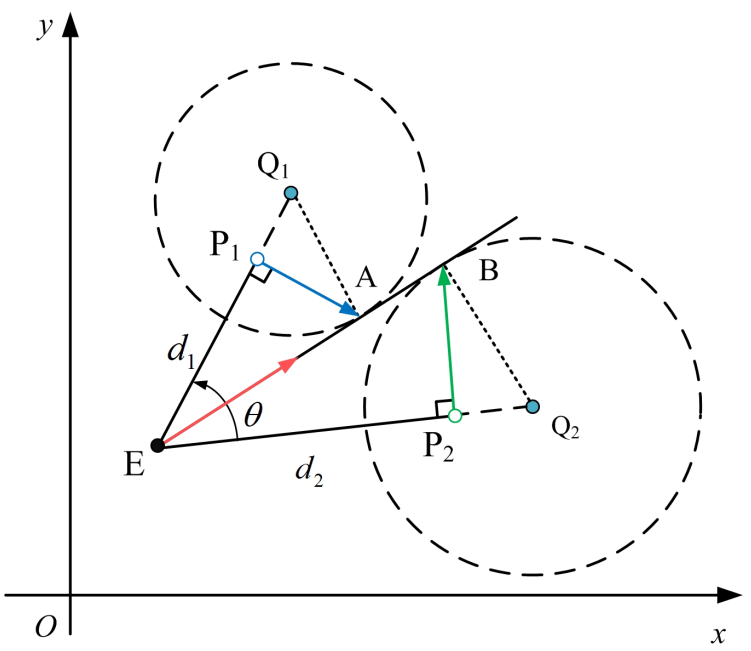

Fig. 4. The optimal strategy of the players when the evader moves along a straight line and $\theta=\arccos \left(1-2 a^{2}\right)$. Since the evader only can go straight, there is no interspace between $A$ and $B$ for the evader to escape.

\section{B. Competition on the Distance $\min \left\{d_{1}, d_{2}\right\}$}

We now study the second sub-game of kind in which the termination conditions are that: when $\min \left\{d_{1}, d_{2}\right\}=0$, the pursuers win, when $\min \left\{d_{1}, d_{2}\right\}>0$ and $\theta=\pi$, the evader wins. In fact, when the initial state lies in the region $\Omega \backslash\left\{\mathscr{D}_{p}^{1} \cup \mathscr{D}_{e}^{1}\right\}$, if the target of the pursuers, $\min \left\{d_{1}, d_{2}\right\}=0$, is unreachable, the evader will win the game because it has a faster speed and can always choose a strategy according to (9) so as to $\dot{\theta}>0$ no matter what strategies of the pursuers. This implies that $\min \left\{d_{1}, d_{2}\right\}=0$ and $\min \left\{d_{1}, d_{2}\right\}>0$ are complementary, and consequently we can use Isaacs's approach [7] to construct the corresponding barrier.

However, it is very difficult to obtain the analytical form of the barrier based on the reduced state equations $(4) \sim(6)$, since the retrogressive path equations (RPEs) along with them is quite complicated to solve. To this end, we start from the state equations $(1) \sim(3)$ and have the following main equation

$$
\begin{aligned}
\min _{\phi_{1}, \phi_{2}} \max _{\varphi} & v_{p}\left(\lambda_{1} \sin \phi_{1}-\lambda_{2} \cos \phi_{1}\right)+v_{p}\left(\lambda_{3} \sin \phi_{2}-\right. \\
& \left.-\lambda_{4} \cos \phi_{2}\right)+v_{e}\left(\lambda_{5} \sin \varphi-\lambda_{6} \cos \varphi\right)=0 .
\end{aligned}
$$

The min and max are furnished in (23) by $\phi_{1}^{*}, \phi_{2}^{*}$ and $\varphi^{*}$ respectively, where

$$
\sin \phi_{1}^{*}=\frac{-\lambda_{1}}{\rho_{1}}, \quad \cos \phi_{1}^{*}=\frac{\lambda_{2}}{\rho_{1}}, \quad \rho_{1}=\sqrt{\lambda_{1}^{2}+\lambda_{2}^{2}}
$$

$$
\begin{aligned}
& \sin \phi_{2}^{*}=\frac{-\lambda_{3}}{\rho_{2}}, \quad \cos \phi_{2}^{*}=\frac{\lambda_{4}}{\rho_{2}}, \quad \rho_{2}=\sqrt{\lambda_{3}^{2}+\lambda_{4}^{2}} \\
& \sin \varphi^{*}=\frac{\lambda_{5}}{\rho_{3}}, \quad \cos \varphi^{*}=\frac{\lambda_{6}}{\rho_{3}}, \quad \rho_{3}=\sqrt{\lambda_{5}^{2}+\lambda_{6}^{2}}
\end{aligned}
$$

so that the main equation (23) can be rewritten as

$$
-v_{p}\left(\rho_{1}+\rho_{2}\right)+v_{e} \rho_{3}=0 .
$$

We are led to the RPEs [7] as follows:

$$
\begin{aligned}
& \stackrel{\circ}{x}_{1}=\frac{v_{p} \lambda_{1}}{\rho_{1}}, \quad \stackrel{\circ}{y}_{1}=\frac{v_{p} \lambda_{2}}{\rho_{1}} \\
& \stackrel{\circ}{x}_{2}=\frac{v_{p} \lambda_{3}}{\rho_{2}}, \quad \stackrel{\circ}{y}_{2}=\frac{v_{p} \lambda_{4}}{\rho_{2}} \\
& \dot{\circ}_{e}=-\frac{v_{p} \lambda_{5}}{\rho_{3}}, \quad \stackrel{\circ}{y}_{e}=-\frac{v_{p} \lambda_{6}}{\rho_{3}} \\
& \grave{\lambda}_{1}=\grave{\lambda}_{2}=\cdots=\grave{\lambda}_{6}=0
\end{aligned}
$$

where ${ }^{\circ}$ denotes the temporal derivative in retrogressive time.

The target of the pursuers is $\min \left\{d_{1}, d_{2}\right\}=0$, but in fact, the distances $d_{1}$ and $d_{2}$ must be equal to zero simultaneously, because if not the evader can do better by maintaining a small distance to the closer pursuer and decreasing its distance to the further pursuer. Thus we can parameterize the boundary of the pursuers' target set by $x_{1}=x_{2}=x_{e}=s_{1}, y_{1}=y_{2}=y_{e}=$ $s_{2}$. The normal vector $\lambda=\left(\lambda_{1}, \lambda_{2}, \ldots, \lambda_{6}\right)$ of this boundary satisfies

$$
\lambda_{1}+\lambda_{3}+\lambda_{5}=0, \quad \lambda_{2}+\lambda_{4}+\lambda_{6}=0 .
$$

Integrating the RPEs $(28) \sim(31)$ from any point on the BUP, we obtain the optimal trajectories of the players as follows

$$
\begin{array}{r}
x_{1}=s_{1}+\frac{v_{p} \lambda_{1}}{\rho_{1}} \tau, \quad y_{1}=s_{2}+\frac{v_{p} \lambda_{2}}{\rho_{1}} \tau \\
x_{2}=s_{1}+\frac{v_{p} \lambda_{3}}{\rho_{2}} \tau, \quad y_{2}=s_{2}+\frac{v_{p} \lambda_{4}}{\rho_{2}} \tau \\
x_{e}=s_{1}+\frac{v_{e}\left(\lambda_{1}+\lambda_{3}\right)}{\rho_{3}} \tau, \quad y_{e}=s_{2}+\frac{v_{e}\left(\lambda_{2}+\lambda_{4}\right)}{\rho_{3}} \tau
\end{array}
$$

where $\tau$ is the reversed time and $\lambda_{1}, \ldots, \lambda_{4}$ are undetermined constants satisfying (27) and (32).

Fig. 5 shows the trajectories of the players associated with the optimal strategies $(24) \sim(26)$ in the second sub-game, where the pursuers $P_{1}, P_{2}$ and the evader $E$ meet at the point $Q\left(s_{1}, s_{2}\right)$ from their initial positions after $\Delta t$ time. We express these trajectories using the reduced state variables $d_{1}, d_{2}$ and $\theta$, and find that the barrier represented by $(33) \sim(35)$ has the following property.

Theorem 4.3: In the second sub-game of kind, the optimal trajectories of the players on the barrier satisfy

$$
d_{1}=d_{2}, \quad \theta=\arccos \left(1-2 a^{2}\right) .
$$

Proof: Applying the sine law and the Pythagorean theorem to the triangles $\triangle E P_{1} P_{2}$ and $\triangle A P_{2} Q$ (see Fig. 5), we have

$$
\begin{aligned}
& v_{e}^{2} \Delta t^{2}=\left(d_{2} \cos \beta-\frac{r_{12}}{2}\right)^{2}+\left(d_{2} \sin \beta+w\right)^{2} \\
& v_{p}^{2} \Delta t^{2}=w^{2}+\frac{r_{12}^{2}}{4}
\end{aligned}
$$




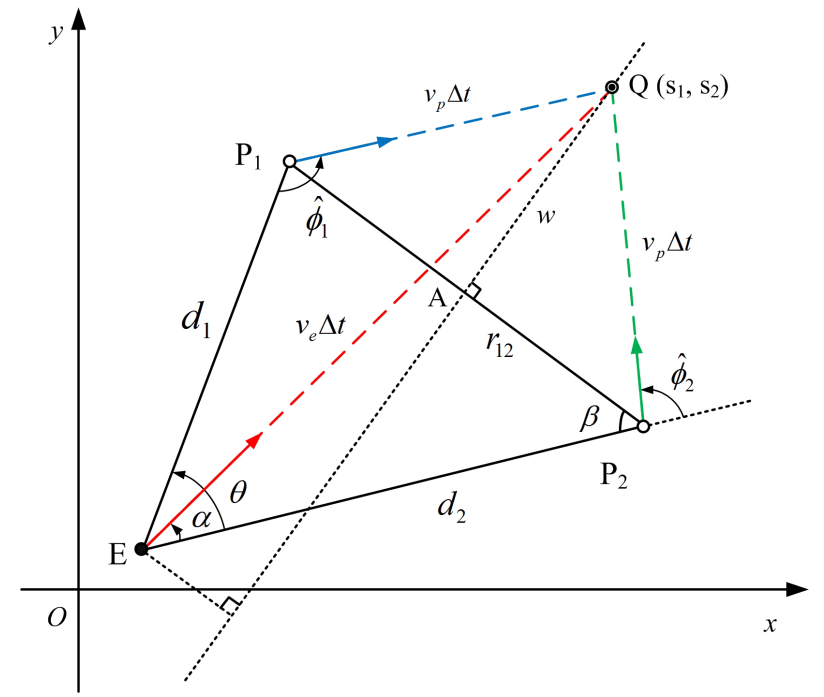

Fig. 5. The trajectories of the players associated with the optimal strategies (24) $\sim(26)$ in the second sub-game, where their destination $Q\left(s_{1}, s_{2}\right)$ is on the perpendicular bisector of line segment $P_{1} P_{2}$. The lengths of segments $A Q, P_{1} Q, E Q$ and $P_{2} Q$ are equal to $w, v_{p} \Delta t, v_{e} \Delta t$ and $v_{p} \Delta t$, respectively. $\beta=\angle E P_{2} P_{1}$.

where

$$
\cos \beta=\frac{d_{2}-d_{1} \cos \theta}{r_{12}}, \quad \sin \beta=\frac{d_{1} \sin \theta}{r_{12}} .
$$

Combining (37) and (38), and eliminating $\Delta t$ yield an equation with respect to $w$,

$$
\begin{array}{r}
\left(1-a^{2}\right) w^{2}-2 a^{2} d_{2} \sin \beta \cdot w+\frac{r_{12}^{2}}{4}\left(1-a^{2}\right)- \\
-a^{2} d_{2}^{2}+a^{2} d_{2} r_{12} \cos \beta=0 .
\end{array}
$$

The pursuers' win requires that the discriminant of equation (40) must be non-negative:

$$
\begin{aligned}
& 4 a^{4} d_{2}^{2} \sin ^{2} \beta-4\left(1-a^{2}\right) . \\
& \cdot\left[\frac{r_{12}^{2}}{4}\left(1-a^{2}\right)-a^{2} d_{2}^{2}+a^{2} d_{2} r_{12} \cos \beta\right] \geq 0 .
\end{aligned}
$$

Simplifying the above inequality

$$
4 a^{2} d_{2}^{2}-\left[\left(1-a^{2}\right) r_{12}+2 a^{2} d_{2} \cos \beta\right]^{2} \geq 0
$$

then substituting (39) into (41), we see that

$$
r_{12} \leq a\left(d_{1}+d_{2}\right)
$$

On the contrary, the evader's win demands that equation (40) has no real root which implies that $r_{12}>a\left(d_{1}+d_{2}\right)$. Thus the above suggests that $r_{12}=a\left(d_{1}+d_{2}\right)$ holds on the barrier, but substantiation is needed.

When $r_{12}=a\left(d_{1}+d_{2}\right)$ holds, $\theta=\Theta$, the root of equation (40) on the barrier is

$$
w_{0}=\frac{a^{2} d_{2} \sin \beta}{1-a^{2}}=\frac{a d_{1} d_{2} \sin \Theta}{\left(1-a^{2}\right)\left(d_{1}+d_{2}\right)}
$$

where $\Theta$ is the non-escape angle represented by (13). And the corresponding time consumption is given by

$$
\Delta t_{0}^{2}=\frac{d_{1} d_{2}}{v_{e}^{2}-v_{p}^{2}} .
$$

Moreover, it is easy to see that the optimal strategy of the evader satisfies

$$
\cos \alpha^{*}=\frac{\sqrt{1-a^{2}}\left(d_{1}+d_{2}\right)}{2 \sqrt{d_{1} d_{2}}}=\sqrt{\frac{\cos \Theta+1}{2}}
$$

which implies that $\alpha^{*}=\Theta / 2=\theta / 2$. Then according to the sine law, the optimal strategies of the pursuers can be derived by

$$
\begin{aligned}
& \sin \hat{\phi}_{1}^{*}=\frac{\sin \alpha^{*}}{a}, \quad \cos \hat{\phi}_{1}^{*}=\frac{\sqrt{1-a^{2}}\left(d_{1}-d_{2}\right)}{2 a \sqrt{d_{1} d_{2}}} \\
& \sin \hat{\phi}_{2}^{*}=\frac{\sin \alpha^{*}}{a}, \quad \cos \hat{\phi}_{2}^{*}=\frac{\sqrt{1-a^{2}}\left(d_{2}-d_{1}\right)}{2 a \sqrt{d_{1} d_{2}}}
\end{aligned}
$$

and obviously $\hat{\phi}_{1}^{*}+\hat{\phi}_{2}^{*}=\pi$. Thus we see that $\triangle E P_{1} Q$ and $\triangle E P_{2} Q$ in Fig. 5 are congruent triangles, then $d_{1}=d_{2}$. From Remark 4.2 we know that $\theta=\arccos \left(1-2 a^{2}\right)$ at this point.

It can be seen from Theorem 4.3 that the state satisfying (36) is exactly the intersection of the boundaries $\mathscr{B}_{1}$ and $\mathscr{B}_{2}$, which means that the barrier of the whole fishing game is beginning to extend from (36). Next, we will integrate the solutions of the two sub-games of kind based on this point.

\section{Integration of the Winning Regions and the Barrier}

We have known that in the first sub-game $\mathscr{D}_{p}^{1}$ and $\mathscr{D}_{e}^{1}$ are the winning regions of the pursuers and the evader, respectively, and the intersection of their boundaries is the barrier in the second sub-game. Since $\Omega \backslash \mathscr{D}_{p}^{1} \supset \mathscr{D}_{e}^{1}$, it is predictable that the evader can achieve success when the state lies in the region $\Omega \backslash\left\{\mathscr{D}_{p}^{1} \cup \mathscr{D}_{e}^{1}\right\}$ as long as it constantly changes its direction to maximize the included angle $\theta$. Therefore, the pursuers' winning region of the whole game is $\mathscr{D}_{p}^{1}$ while the evader's winning region is $\Omega \backslash \mathscr{D}_{p}^{1}$, thus the barrier of the whole game is given by $\mathscr{B}_{1}$.

In order to demonstrate that the barrier actually has the above characteristics, it is convenient to switch to a threedimensional relative state space [16], where the state variables are $x, y$ and $z$ (see Fig. 6). The coordinates of the pursuers and the evader in the plane correspond to $P_{1}(0, z), P_{2}(0,-z)$ and $E(x, y)$, respectively.

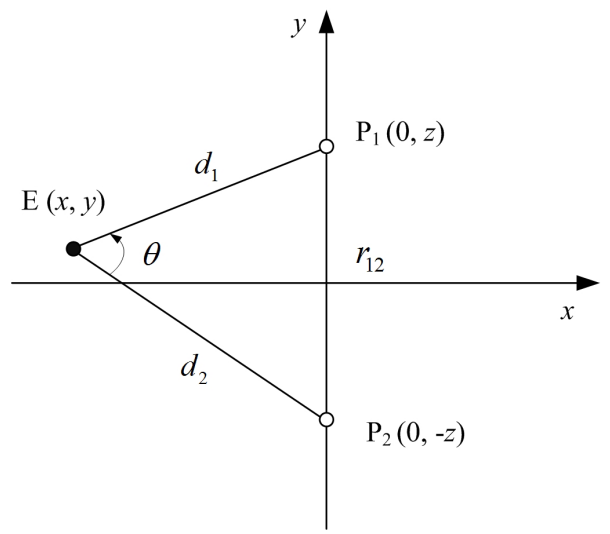

Fig. 6. Fishing game in the relative state space with variables $x, y$ and $z$. 
In Fig. 7, the barrier of fishing game in the threedimensional relative state space are shown. From initial points above the barrier, the evader can choose a suitable strategy to succeed in passing the gap between the two pursuers. On the contrary, from points below the barrier, the two pursuers can adopt suitable strategies to prevent the evader's threading successfully.

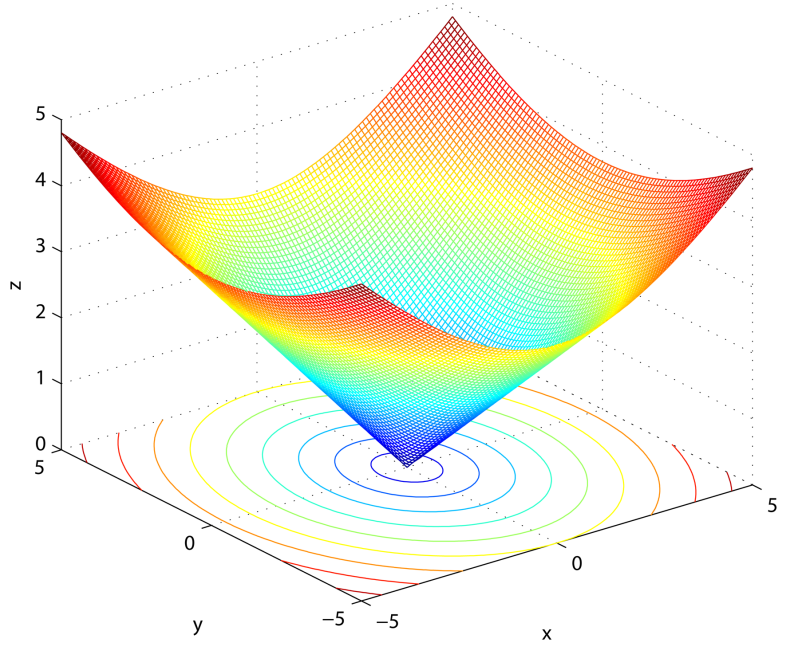

Fig. 7. The barrier of fishing game in the three-dimensional relative state space with $a=0.6$.

A more intuitive delineation of the barriers with different $a$ and a fixed $z$ is sketched in Fig. 8, where the locus of $E$ conforming to the surface $\mathscr{B}_{1}$ is an ellipse, which is given by the following equation

$$
x^{2}+\left(1-a^{2}\right) y^{2}=\frac{\left(1-a^{2}\right) z^{2}}{a^{2}} .
$$

The inner side of the ellipse (excluding the points $P_{1}$ and $P_{2}$ ) is the escape zone, whose size decreases with $a$.

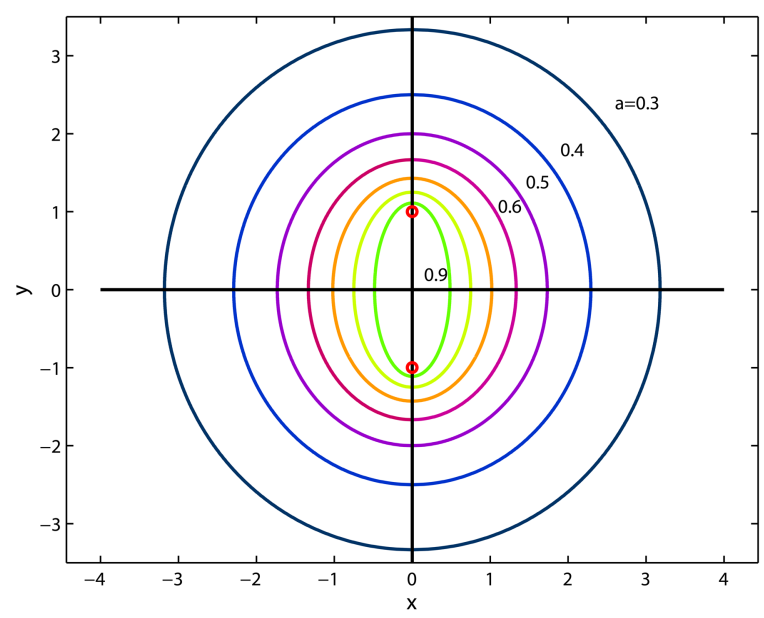

Fig. 8. The barriers of fishing game with a fixed $z=1$ for different $a$ (from 0.9 to 0.3 ), where the two small red circles represent the positions of the pursuers.

\section{Optimal Strategies For the Players}

We have already constructed the forms of the barrier and winning regions analytically in the fishing game, which comprise a solution in the game of kind. In this section, we will discuss the optimal strategies for the players in scope of a game of degree [7]. This work, fusion of the games of kind and degree, contains of two parts: when the initial state lies in $\mathscr{D}_{p}^{1}$, the objective of the pursuers will be to capture the evader as effectively as possible, further minimizing their winning time $T_{1}$. On the contrary, the evader will endeavor to delay the pursuers' victory to maximize the time $T_{1}$. When the initial state is in $\Omega \backslash \mathscr{D}_{p}^{1}$, the objective of the pursuers will be maximizing the evader's winning time $T_{2}$ which the evader seeks to minimize ${ }^{1}$. Similar to the previous situation encountered in the game of kind, the main obstruction in the game of degree is also the characterization of the competition focuses, $\theta$ and $\min \left\{d_{1}, d_{2}\right\}$. Thus we continue to use the method of explicit policy, partitioning the state space into several regions and then solving the optimal strategies of the players in these regions respectively.

\section{A. Optimal Strategies in the Pursuers' Winning Region}

We take the winning time $T_{1}$ or $T_{2}$ as the payoff of the fishing game of degree. Thus, when the initial positions of the players are in the pursuers' winning region $\mathscr{D}_{p}^{1}$, the payoff function is given by

$$
\min _{\hat{\phi}_{1}, \hat{\phi}_{2}} \max _{\alpha} J_{1}=\min _{\hat{\phi}_{1}, \hat{\phi}_{2}} \max _{\alpha} \int_{t_{0}}^{T_{1}} 1 d t
$$

Naturally, if the evader starts from the pursuers far enough, as shown in Fig. 9, the two pursuers can go straight towards each other to prevent the evader from threading, while the evader only can move towards the midpoint $Q$ between the initial positions of the pursuers as close as possible.

According to the sine law and the Pythagorean theorem,

$$
\begin{aligned}
|E Q| & =\sqrt{\left(\frac{1}{2} d_{1} \sin \theta\right)^{2}+\left[d_{1} \cos \theta+\frac{1}{2}\left(d_{2}-d_{1} \cos \theta\right)\right]^{2}} \\
& =\frac{1}{2} \sqrt{d_{1}^{2}+d_{2}^{2}+2 d_{1} d_{2} \cos \theta}
\end{aligned}
$$

then the optimal strategies of the players are

$$
\begin{aligned}
& \sin \hat{\phi}_{1}{ }^{*}=\frac{d_{2} \sin \theta}{r_{12}}, \quad \cos \hat{\phi}_{1}{ }^{*}=\frac{d_{1}-d_{2} \cos \theta}{r_{12}} \\
& \sin \hat{\phi}_{2}{ }^{*}=\frac{d_{1} \sin \theta}{r_{12}}, \quad \cos \hat{\phi}_{2}{ }^{*}=\frac{d_{1} \cos \theta-d_{2}}{r_{12}} \\
& \sin \alpha^{*}=\frac{\frac{1}{2} d_{1} \sin \theta}{|E Q|}=\frac{d_{1} \sin \theta}{\sqrt{d_{1}^{2}+d_{2}^{2}+2 d_{1} d_{2} \cos \theta}} \\
& \cos \alpha^{*}=\frac{\frac{1}{2}\left(d_{2}+d_{1} \cos \theta\right)}{|E Q|}=\frac{d_{2}+d_{1} \cos \theta}{\sqrt{d_{1}^{2}+d_{2}^{2}+2 d_{1} d_{2} \cos \theta}}
\end{aligned}
$$

${ }^{1}$ Note that the objective of the pursuers is not the traditional pattern of minimizing the closest distance to the evader in this case, because it will not only lead to the loss of their original superiority of cooperation but also increase the evader's advantage in speed. 
However, the pursuers' strategies (46) and (47) are available only when

$$
\frac{r_{12}}{2 v_{p}} \leq \frac{|E Q|}{v_{e}}
$$

that is,

$$
\frac{r_{12}^{2}}{4 v_{p}^{2}} \leq \frac{d_{1}^{2}+d_{2}^{2}+2 d_{1} d_{2} \cos \theta}{4 v_{e}^{2}}=\frac{2\left(d_{1}^{2}+d_{2}^{2}\right)-r_{12}^{2}}{4 v_{e}^{2}}
$$

Simplifying the above inequality, we have the following conditions with respect to the state variables

$$
r_{12}^{2} \leq \frac{2 a^{2}}{1+a^{2}}\left(d_{1}^{2}+d_{2}^{2}\right)
$$

or

$$
\cos \theta \geq \frac{\left(1-a^{2}\right)\left(d_{1}^{2}+d_{2}^{2}\right)}{2\left(1+a^{2}\right) d_{1} d_{2}} .
$$

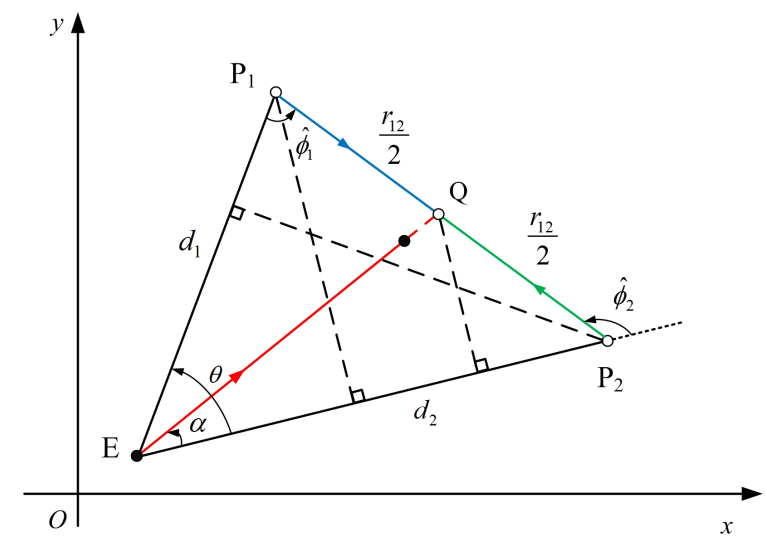

Fig. 9. The optimal trajectories of the players when the state satisfies the condition (53), where $Q$ is the midpoint of segment $P_{1} P_{2}$.

Corresponding to the previous three-dimensional relative state space, the locus of $E(x, y)$ on the boundary of (53) denoted by $\mathscr{B}_{3}$ is a circle

$$
x^{2}+y^{2}=\frac{z^{2}}{a^{2}}
$$

and the terminal time $T_{1}=z\left(t_{0}\right) / v_{p}+t_{0}$.

Additionally, for the boundary $\mathscr{B}_{2}$, the locus of $E$ is a configuration of two segments of the circular arcs

$$
\left(x \pm \frac{\left(1-2 a^{2}\right) z}{2 a \sqrt{1-a^{2}}}\right)^{2}+y^{2}=\frac{z^{2}}{4 a^{2}\left(1-a^{2}\right)}
$$

where the notation " \pm " will be " + " when $x \leq 0$, or " - " when $x>0$, and the intersections of the two circular arcs with the $x$-axis must be at $\left( \pm z \sqrt{1-a^{2}} / a, 0\right)$. Particularly, when $a=$ $\sqrt{2} / 2, \mathscr{B}_{2}$ will be a perfect circle furnished by $x^{2}+y^{2}=z^{2}$.

Thus we can partition the relative state space into four zones using the boundaries $\mathscr{B}_{1}, \mathscr{B}_{2}$, and $\mathscr{B}_{3}$, such as an instance shown in Fig. 10 for a given $z$, where zone 1 and zone 2 including $\mathscr{B}_{3}$ and $\mathscr{B}_{1}$ constitute the pursuers' winning region $\mathscr{D}_{p}^{1}$, while zone 3 and zone 4 excluding the points $P_{1}, P_{2}$ constitute the evader's winning region $\Omega \backslash \mathscr{D}_{p}^{1}$.

In particular, from Fig. 10 we can see that $\mathscr{B}_{1}$ is tangent to $\mathscr{B}_{3}$ at the point with $\left\{r_{12}=a\left(d_{1}+d_{2}\right)\right.$, and $\left.\theta=0\right\}$, which inspires us that after the state holds on $\left\{r_{12} \leq a\left(d_{1}+\right.\right.$

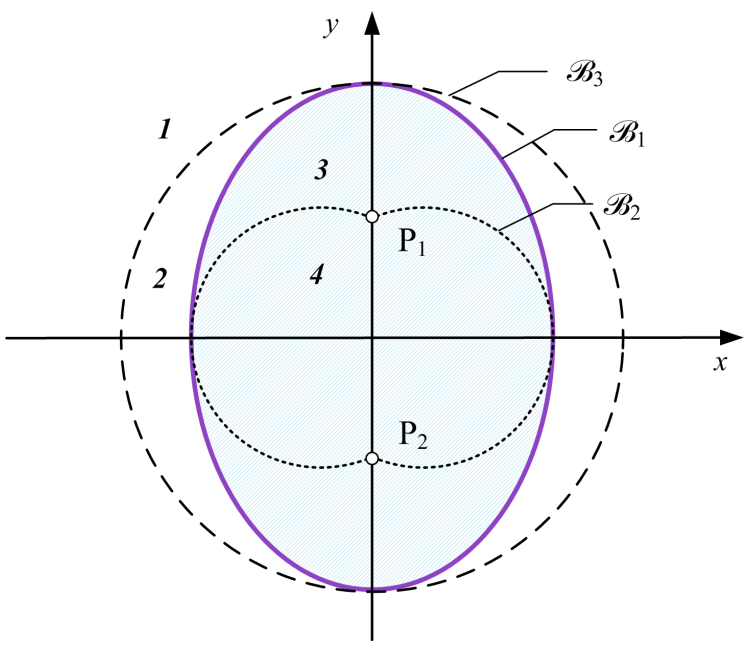

Fig. 10. The partition of the relative state space, where the shaded region is the evader's winning region.

$d_{2}$ ), and $\left.\theta=0\right\}$, the pursuers can adopt the strategies (46) and (47) to achieve rendezvous, i.e., $r_{12}=0$, and then terminate the game (responding to Remark 4.3).

Thus in zone 2 with the boundary $\mathscr{B}_{1}$, the pursuers will choose some suitable strategies to decrease the included angle $\theta$ to zero as quickly as possible. Obviously, the strategies represented by (8) are optimal, and correspondingly the evader has to choose the strategy (9) to maximize the pursuers' winning time $T_{1}$. Their trajectories associated with the optimal strategies are shown in Fig. 11.

When $d_{1}^{0}<d_{2}^{0}$, the evader $E$ tends to $P_{2}$ with curvilinear motion (Fig. 11(a)). When $d_{1}^{0}=d_{2}^{0}$, the evader moves straight along with the angular bisector of $\theta_{0}$, and correspondingly the pursuers have two options: one is using strategy (8) in curvilinear motion (see Fig. 11 (b)), the other one is choosing strategies (24) and (25) to directly meet the evader at a point (see Fig. 11 (c)). Comparatively speaking, the latter is better than the former because of less time consumption, but if the evader does not play optimally, the former will be better since (8) is an optimal feedback strategy while (24) and (25) are the conservative open-loop strategies only depending on the initial state.

Certainly, the trajectories generated from the above two options coincide with each other when $d_{1}^{0}=d_{2}^{0}$ and $\theta_{0}=$ $\arccos \left(1-2 a^{2}\right)$ (see Fig. 11 (d)). From (42), we know that the time consumption at this point, is $d_{1}^{0} / \sqrt{v_{e}^{2}-v_{p}^{2}}$. Thus in zone 2, for the situations (c) and (d) the terminal time satisfies $T_{1} \leq t_{0}+d_{1}^{0} / \sqrt{v_{e}^{2}-v_{p}^{2}}$

Suppose the final values of $d_{1}, d_{2}$ and $r_{12}$ are equal to $d_{1}^{f}, d_{2}^{f}$ and $r_{12}^{f}$ when the game terminates, respectively. For the situation (b), since $\theta_{0}<\arccos \left(1-2 a^{2}\right), \cos \alpha^{*}=$ $\cos \left(\theta_{0} / 2\right)>\sqrt{1-a^{2}}$, then from (4) $\sim(5)$ and (8) $\sim(9)$ we know that

$$
\left|\dot{d}_{1}\right|=\left|\dot{d}_{2}\right|>v_{e} \sqrt{1-a^{2}}=\sqrt{v_{e}^{2}-v_{p}^{2}} .
$$




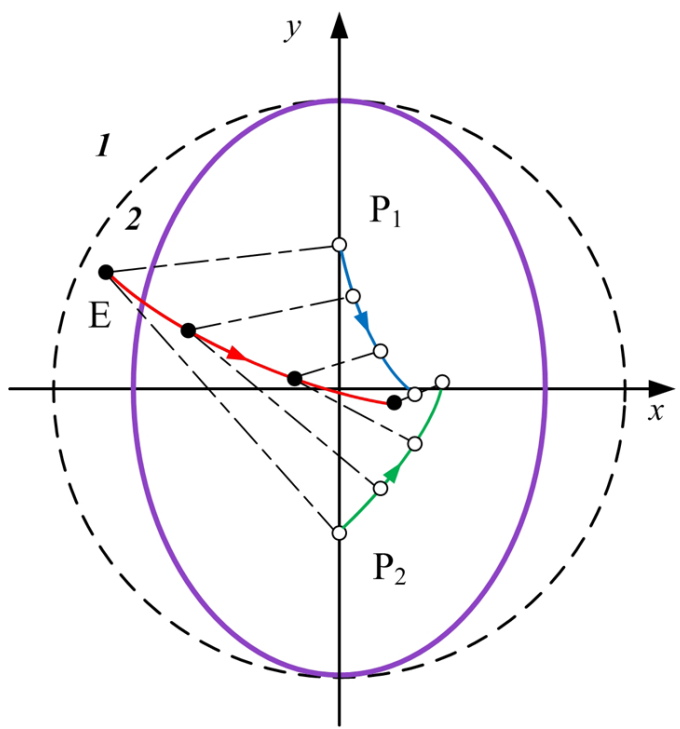

(a) $d_{1}{ }^{0}<d_{2}{ }^{0} \quad \cos \theta_{0}>\cos \Theta_{0}$

$$
0<r_{12}{ }^{f}<a\left(d_{1}^{f}+d_{2}^{f}\right)
$$

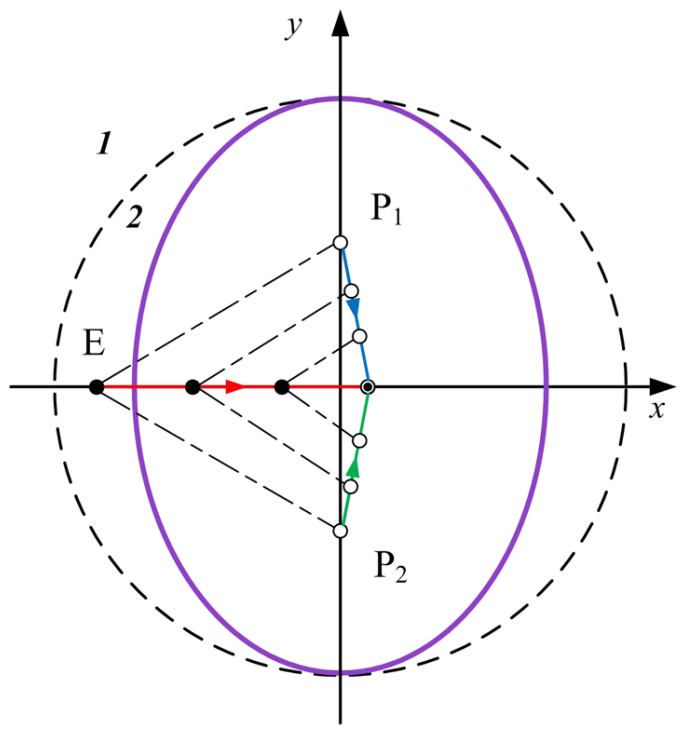

(c) $d_{1}{ }^{0}=d_{2}{ }^{0} \cos \theta_{0}>\cos \Theta_{0}$

$$
d_{1}^{f}=d_{2}{ }^{f}=r_{12}{ }^{f}=0
$$

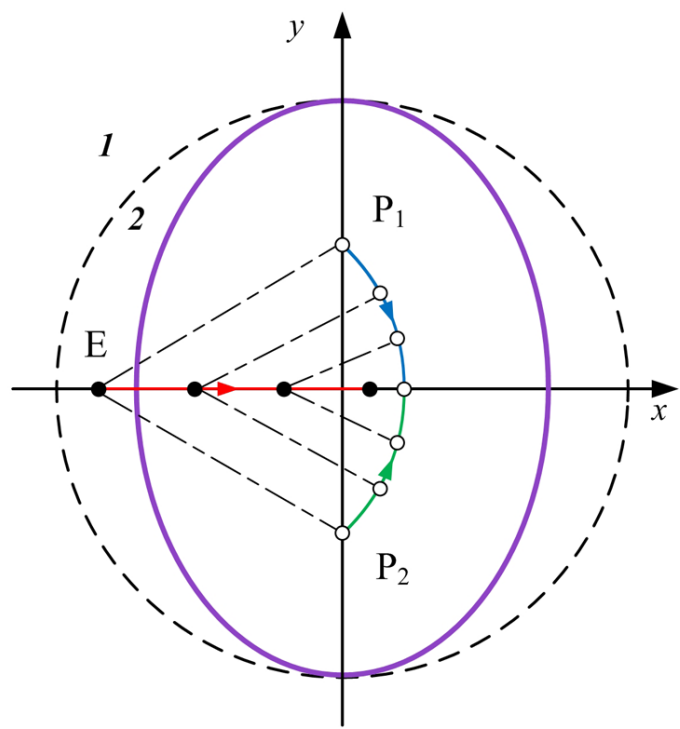

(b) $\quad d_{1}^{0}=d_{2}^{0} \quad \cos \theta_{0}>\cos \Theta_{0}$

$d_{1}^{f}=d_{2}{ }^{f}>0 \quad r_{12}{ }^{f}=0$

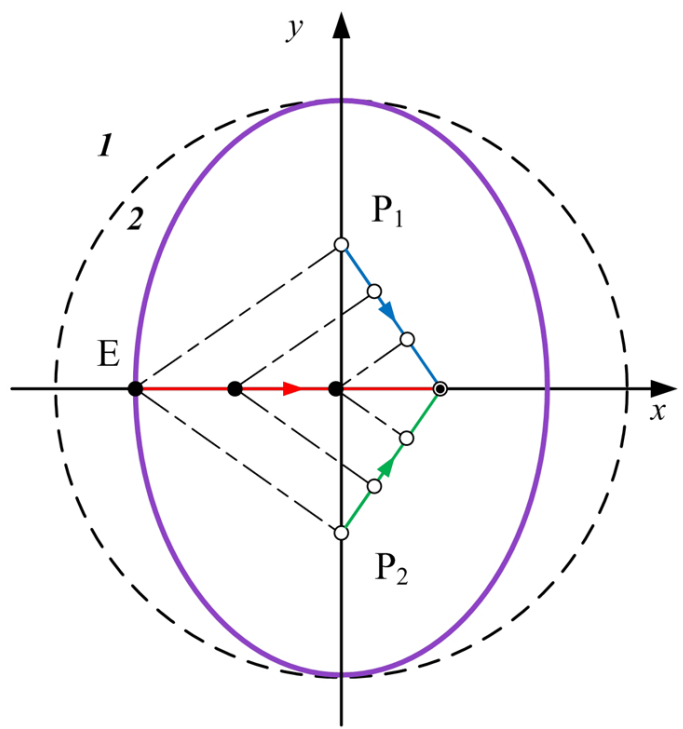

(d) $\quad d_{1}{ }^{0}=d_{2}{ }^{0} \cos \theta_{0}=1-2 a^{2}$

$$
d_{1}^{f}=d_{2}{ }^{f}=r_{12}{ }^{f}=0
$$

Fig. 11. The optimal trajectories of the players in zone 2 , where $d_{1}^{f}, d_{2}^{f}$ and $r_{12}^{f}$ are the final values of $d_{1}, d_{2}$ and $r_{12}$ when the game terminates, respectively.

Thus the terminal time

$$
T_{1}<t_{0}+\frac{d_{1}^{0}-d_{1}^{f}}{\sqrt{v_{e}^{2}-v_{p}^{2}}} \leq t_{0}+\frac{d_{1}^{0}}{\sqrt{v_{e}^{2}-v_{p}^{2}}} .
$$

Similarly, for the situation (a) with $d_{1}^{0}<d_{2}^{0}$, since in the final time the value of $z$ satisfies

$$
z_{f}=\frac{r_{12}^{f}}{2}<\frac{a\left(d_{1}^{f}+d_{2}^{f}\right)}{2}<a d_{2}^{f}
$$

then according to the discussion of terminal time in zone 1 ,

$$
\begin{aligned}
T_{1} & <t_{0}+\frac{d_{2}^{0}-d_{2}^{f}}{\sqrt{v_{e}^{2}-v_{p}^{2}}}+\frac{a d_{2}^{f}}{v_{p}} \\
& <t_{0}+\frac{d_{2}^{0}-d_{2}^{f}}{\sqrt{v_{e}^{2}-v_{p}^{2}}}+\frac{d_{2}^{f}}{\sqrt{v_{e}^{2}-v_{p}^{2}}} \\
& =t_{0}+\frac{d_{2}^{0}}{\sqrt{v_{e}^{2}-v_{p}^{2}}}
\end{aligned}
$$


In a word, the terminal time $T_{1}$ in zone 2 satisfies

$$
T_{1} \leq t_{0}+\frac{\max \left\{d_{1}^{0}, d_{2}^{0}\right\}}{\sqrt{v_{e}^{2}-v_{p}^{2}}} .
$$

\section{B. Optimal Strategies in the Evader's Winning Region}

When the initial positions of the players lie in the evader's winning region $\Omega \backslash \mathscr{D}_{p}^{1}$, the payoff function of the fishing game of degree is changed into

$$
\max _{\hat{\phi}_{1}, \hat{\phi}_{2}} \min _{\alpha} J_{2}=\max _{\hat{\phi}_{1}, \hat{\phi}_{2}} \min _{\alpha} \int_{t_{0}}^{T_{2}} 1 d t
$$

where $T_{2}$ is the evader's winning time.

Symmetric with the case in zone 2, the optimal strategies of the pursuers and the evader in zone 3 are given by (8) and (9) respectively. The corresponding optimal trajectories of the players are shown in Fig. 12, where we can see that they travel on spirals maintaining the rate of their relative distance, $b=d_{1} / d_{2}$, constant and equal to the initial value $b_{0}=d_{1}^{0} / d_{2}^{0}$. Similarly, the terminal time satisfies $T_{2} \leq$ $t_{0}+\max \left\{d_{1}^{0}, d_{2}^{0}\right\} / \sqrt{v_{e}^{2}-v_{p}^{2}}$ since $\theta$ will increase to $\pi$ prior to that $\min \left\{d_{1}, d_{2}\right\}$ decreasing to zero.

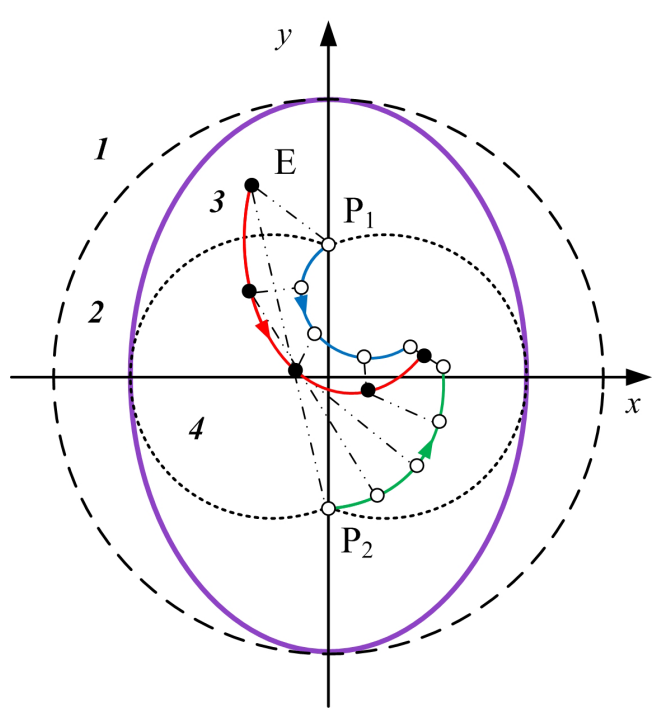

Fig. 12. The optimal trajectories of the players in zone 3.

When the initial state lies in zone 4, clearly, from the proofs of Theorem 4.2, we know that the evader will succeed as long as it moves straight towards the interspace between two Apollonius circles (as shown in Fig. 3). Thus, the optimal strategy of the evader satisfies $\xi<\alpha^{*}<\theta-\xi(\xi=\arcsin a)$. Correspondingly, the optimal trajectories of the pursuers will also be the straight lines. Suppose the evader moves to $E^{\prime}$ in $\Delta t$ time with the heading $\alpha$, in order to maximize the terminal time $T_{2}$ the pursuers $P_{1}$ and $P_{2}$ should select $P_{1}^{\prime}$ and $P_{2}^{\prime}$ as their endpoints, respectively, to minimize the possible included angle $\theta^{\prime}$ (see Fig. 13 (a), next page). Thus at the terminal time $T_{2}$, Fig. 13 (b) will be the final situation of the fishing game, where the optimal strategies (headings) of the pursuers are perpendicular to line $P_{1} P_{2}$ respectively. Then the expression of $\Delta t$ can be derived according to the sine law,

$$
\Delta t=\frac{d_{1} d_{2} \sin \theta}{r_{12}\left(v_{e} \sin (\alpha+\beta)-v_{p}\right)}
$$

where $\sin \beta=d_{1} \sin \theta / r_{12}, \cos \beta=\left(d_{2}-d_{1} \cos \theta\right) / r_{12}$. For the evader, to minimize $\Delta t$, the value of $\alpha^{*}$ within the interval $(\xi, \theta-\xi)$ should furnish the $\max$ in $\sin (\alpha+\beta)$. Thus the terminal time $T_{2}$ satisfies

$$
T_{2} \leq t_{0}+\frac{d_{1}^{0} d_{2}^{0} \sin \theta_{0}}{r_{12}^{0}\left(v_{e} \min \left\{\sigma_{1}, \sigma_{2}\right\}-v_{p}\right)}
$$

where

$$
\begin{aligned}
& \sigma_{1}=\frac{1}{r_{12}^{0}}\left[a\left(d_{2}^{0}-d_{1}^{0} \cos \theta_{0}\right)+\sqrt{1-a^{2}} d_{1}^{0} \sin \theta_{0}\right] \\
& \sigma_{2}=\frac{1}{r_{12}^{0}}\left[a\left(d_{1}^{0}-d_{2}^{0} \cos \theta_{0}\right)+\sqrt{1-a^{2}} d_{2}^{0} \sin \theta_{0}\right] .
\end{aligned}
$$

In particular, if $d_{1}^{0}=d_{2}^{0}$, then $\alpha^{*}=\theta_{0} / 2$ and $T_{2}$ reaches the minimum $t_{0}+\frac{d_{1}^{0} \sin \theta_{0}}{\sqrt{2+2 \cos \theta_{0}}\left(v_{e}-v_{p}\right)}$.

\section{Conclusions}

A so-called "fishing game" with point capture is investigated in this paper. Due to the faster speed of the evader, the possibility of capture or escape is required to be determined first. A critical aspect in the game of kind, barrier, is constructed analytically by using the method of explicit policy, which shows that the pursuers or the evader can adopt some suitable strategies to achieve success when the initial state lies in their own winning regions. Then for these winning regions, the players' optimal feedback strategies are solved in terms of the game of degree. Furthermore, corresponding to the four zones of the initial state space, the optimal trajectories of the players are delineated and the upper bounds of their winning times are also derived.

Since general pursuit-evasion game with more than two pursuers and one evader can be regarded as a combination of multiple fishing games, the findings of this paper are useful for analyzing the interception, tracking, besiegement or collision avoidance of multi-robot systems to some faster targets. Especially, the barrier can be served as not only the basis for determining the number of the pursuers, but also a necessary and sufficient condition of successful capture in related pursuit-evasion games with point capture. Besides, the method of explicit policy is computationally efficient in generating control strategies and winning conditions for the players, and avoids solving the complicated HJI equations. With the aid of geometric analysis, the obtained expression of the barrier is fairly concise.

Due to the requirement of passing the gap between two pursuers, the evader's actions are bounded in the fishing game. Next step, we will be interested in studying the game of kind with other restrictions on the players, such as the pursuers' cooperation and the more complex motion models (e.g. the motion model of robotic fish [60]). The pursuit-evasion game with more pursuers is another focus forward in the direction of our research. 


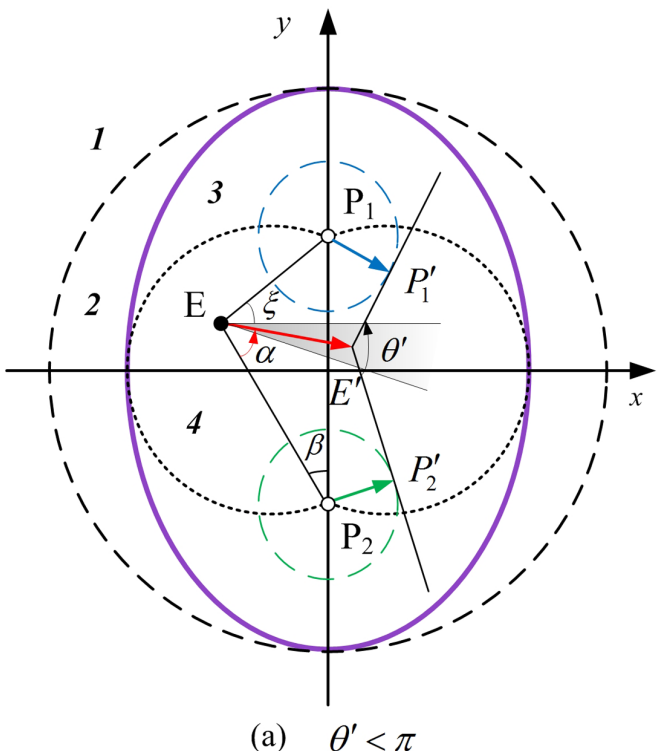

(a) $\theta^{\prime}<\pi$

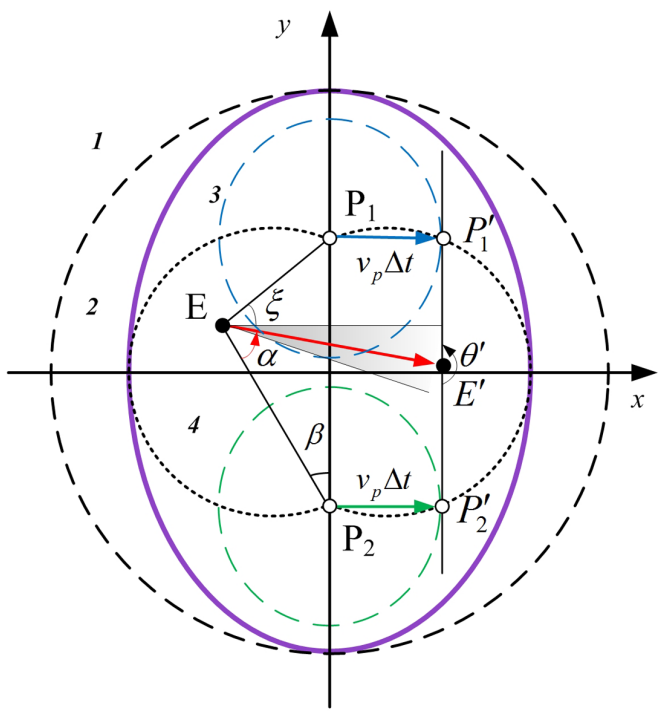

(b) $\theta^{\prime}=\pi$

Fig. 13. The optimal trajectories of the players in zone 4, where the blue (green) dashed circle represents the attainable location of $P_{1}\left(P_{2}\right)$ after $\Delta t$ time, and the shaded area is the interspace between two Apollonius circles associated with the initial positions of the players.

\section{REFERENCES}

[1] H. Huang, J. Ding, W. Zhang, and C. J. Tomlin, "Automation-assisted capture-the-flag: a differential game approach," IEEE Trans. Control Syst. Technol., vol. 23, no. 3, pp. 1014-1028, Oct. 2014.

[2] X. Wang and H. Duan, "Biologically adaptive robust mean shift algorithm with Cauchy predator-prey BBO and space variant resolution for unmanned helicopter formation," Sci. China Inf. Sci., vol. 57, no. 11, pp. 1-13, Nov. 2014.

[3] G. C. Chasparis and J. S. Shamma, "Network formation: neighborhood structure, establishment costs, and distributed learning," IEEE Trans. Cybern., vol. 43, no. 6, pp. 1950-1962, Dec. 2013.

[4] T. Oral and F. Polat, "MOD* Lite: an incremental path planning algorithm taking care of multiple objectives," IEEE Trans. Cybern., vol. 46, no. 1, pp. 245-257, Jan. 2016.

[5] K. Fregene, D. C. Kennedy, and D. W. L. Wang, "Toward a systems- and control-oriented agent framework," IEEE Trans. Syst., Man, Cybern. B, Cybern., vol. 35, no. 5, pp. 999-1012, Oct. 2005.

[6] H. Li, H. Wang, and J. Liu, "Error aware multiple vertical planes based visual localization for mobile robots in urban environments," Sci. China Inf. Sci., vol. 58, no. 3, pp. 1-14, Mar. 2015.

[7] R. Isaacs, Differential Games. New York: John Wiley, 1965.

[8] T. Başar and G. J. Olsder, Dynamic Noncooperative Game Theory, 2nd ed., Philadelphia, PA, USA: SIAM, 1999.

[9] A. W. Merz, "The Homicidal Chauffeur- A Differential Game," PhD thesis, Stanford Univ., CA, USA, 1971.

[10] V. S. Patsko and V. L. Turova, "Families of semipermeable in differential games with the Homicidal Chauffeur dynamics," Automatica, vol. 40, pp. 2059-2068, Sep. 2004.

[11] J. Lewin and J. V. Breakwell, "The surveillance evasion game of degree," J. Optim. Theory Appl., vol. 16, nos. 3/4, pp. 339-353, Aug. 1975.

[12] M. S. David, "Policies and controller design for a pursing vehicle," IEEE Trans. Autom. Control, vol. AC-14, no. 5, pp. 482-488, Oct. 1969.

[13] V. S. Patsko and V. L. Turova, Homicidal Chauffeur Game: History and Modern Studies, Scientific report. Ekaterinburg: Russian Academy of Sciences, 2009.

[14] I. Exarchos, P. Tsiotras, and M. Pachter, "On the Suicidal Pedestrain differential game," Dyn. Games Appl., Published online, Dec. 2014.

[15] J. V. Breakwell, "Pursuit of a faster evader," in The Theory and Application of Differential Games, J. D. Grote Ed. Coventry, England, pp. 243-256, Aug. 1974.

[16] P. Hagedorn and J. V. Breakwell, "A differential game with two pursuers and one evader," J. Optim. Theory Appl., vo. 18, no. 1, pp. 15-29, Jan. 1976.

[17] A. W. Merz, "The game of two identical cars," J. Optim. Theory Appl., vol. 9 , no. 5, 324-343, 1972.
[18] M. Pachter and W. M. Getz, "The geometry of the barrier in the 'game of two cars'," Optim. Control Appl. Met., vol. 1, pp. 103-118, Apr. 1980.

[19] W. M. Getz and M. Pachter, "Capturability in a two-target 'game of two cars'," J. Guid. Control, vol. 4, no. 1, pp. 15-21, Jan.-Feb. 1981.

[20] F. Imado, "Pursuit-evasion problems of two cars in an ellipsoid under gravity," Eng. Optim., vol. 43, no. 4, pp. 447-465, Apr. 2011.

[21] M. Zhang and H. H. Liu, "Game theoretical persistent tracking of a moving target using a unicycle-type mobile vehicle," IEEE Trans. Ind. Electron., vol. 61, no. 11, pp. 6222-6233, Apr. 2014.

[22] P. Chen and S. Sastry, "Pursuit controller performance guarantees for a lifeline pursuit-evasion game over a wireless sensor network," in Proc. 45th IEEE Conf. Decision Control, 2006, pp. 691-696.

[23] A. A. Galyaev and E. P. Maslov, "Spatial evasion of the rotating detection zone. I," Automat. Rem. Control, vol. 74, no. 7, pp. 1178-1190, 2013.

[24] A. A. Galyaev and E. P. Maslov, "Evading a rotating detection zone on a plane," J. Comput. Syst. Sci. Int., vol. 52, no. 3, pp. 377-385, 2013.

[25] S. Bhattacharya, S. Hutchinson, and T. Basar, "Game-theoretic analysis of a visibility based pursuit-evasion game in the presence of obstacles," in Proc. Amer. Control Conf., 2009, pp. 373-378.

[26] S. Bhattacharya and S. Hutchinson, "On the existence of Nash equilibrium for a two-player pursuit-evasion game with visibility constraints," Int. J. Robot. Res., vol. 29, no. 7, pp. 831-839, Jun. 2010.

[27] S. Bhattacharya and S. Hutchinson, "A cell decomposition approach to visibility-based pursuit evasion among obstacles," Int. J. Robot. Res., vol. 30, no. 14, pp. 1709-1727, Dec. 2011.

[28] S. Bhattacharya, T. Basar, and N. Hovakimyan, "Singular surfaces in multi-agent connectivity maintenance games," in Proc. IEEE Conf. Decision Control Euro. Control Conf., 2011, pp. 261-266.

[29] S. Bhattacharya, T. Başar, and N. Hovakimyan, "On the construction of barrier in a visibility based pursuit evasion game," in Euro. Control Conf., 2014, pp. 1894-1901.

[30] J. Shinar and D. Steinberg, "Analysis of optimal evasive maneuvers based on a linearized two-dimensional kinematic model," J. Aircraft, vol. 14, no. 8, pp. 795-802, Aug. 1977.

[31] N. Farber and J. Shinar, "Approximate solution of singularly perturbed nonlinear pursuit-evasion games," J. Optim. Theory Appl., vol. 32, no. 1, pp. 39-73, Sep. 1980

[32] J. Shinar, M. Medinah, and M. Biton, "Singular surfaces in a linear pursuit-evasion game with elliptical vectograms," J. Optim. Theory Appl., vol. 43, no. 3, pp. 431-456, Jul. 1984.

[33] J. Shinar, M. Guelman, and A. Green, "An optimal guidance law for a planar pursuit-evasion game of kind," Comput. Math. Appl., vol. 18, no. $1-3$, pp. $35-44,1989$.

[34] J. Shinar, V. Y. Glizer, and V. Turetsky, "Robust pursuit of a hybrid evader," Appl. Math. Comput., vol. 217, pp. 1231-1245, 2010. 
[35] S. Bhattacharya, "On the construction of barrier in a connectivity maintenance game," in Euro. Control Conf., 2013, pp. 3338-3345.

[36] Z. Zhou, R. Takei, H. Huang, and C. J. Tomlin, "A general, open-loop formulation for reach-avoid games," in IEEE Conf. Decision Control, 2012, pp. 6501-6506.

[37] M. Chen, Z. Zhou, and C. J. Tomlin, "Multiplayer reach-avoid games via low dimensional solutions and maximum matching," in Amer. Control Conf., 2014, pp. 1444-1449.

[38] R. Takei, H. Huang, J. Ding, and C. J. Tomlin, "Time-optimal multi-stage motion planning with guaranteed collision avoidance via an open-loop game formulation," in IEEE Int. Conf. Robot. Autom., 2012, pp. 323-329.

[39] V. Turetsky, "Capture zone of linear feedback pursuer strategies," Automatica, vol. 44, pp. 560-566, 2008.

[40] H. Huang, W. Zhang, J. Ding, D. M. Stipanović, and C. J. Tomlin, "Guaranteed decentralized pursuit-evasion in the plane with multiple pursuers," in Proc. 50th IEEE Conf. Decision Control Euro. Control Conf., 2011, pp. 4835-4840.

[41] S. Pan, H. Huang, J. Ding, W. Zhang, D. M. Stipanović, and C. J. Tomlin, "Pursuit, evasion and defense in the plane," in Proc. Amer. Control Conf., 2012, pp. 4167-4173.

[42] H. Huang, Z. Zhou, W. Zhang, J. Ding, D. M. Stipanović, and C. J. Tomlin, "Safe-reachable area cooperative pursuit," Adv. Neural Inf. Proc. Syst., vol. 1, pp. 1637-1645, 2013.

[43] S. Alexander, R. Bishop, and R. Ghrist, "Capture pursuit games on unbounded domains," L'Enseignement Mathématique, vol. 55, no. 1 , pp. 103-125, 2009.

[44] T. Kim and T. Sugie, "Cooperative control for target-capturing task based on a cyclic pursuit strategy," Automatica, vol. 43, no. 8, pp. 1426-1431, Aug. 2007.

[45] S. D. Bopardikar, F. Bullo, and J. P. Hespanha, "A cooperative Homicidal Chauffeur game,"Automatica, vol. 45, no. 7, pp. 1771-1777, Jul. 2009.

[46] S. D. Bopardikar and S. Suri, " $k$-Capture in multi-agent pursuit evasion, or the lion and the hyenas," Theor. Comput. Sci., vol. 522, pp. 13-23, 2014.

[47] A. B. Özgüler and A. Yıldız, "Foraging swarms as Nash equilibria of dynamic games," IEEE Trans. Cybern., vol. 44, no. 6, pp. 979-987, Jun. 2014.

[48] G. Barles, Solutions de viscosité des équations de Hamilton-Jacobi. France: Springer-Verlag, 1994.

[49] P. Bernhard, S. Crepey, and A. Rapaport, "Comparison of two numerical approaches for barrier and value of a simple pursuit-evasion game," in Adv. Dyn. Games Appl.(Annals of the International Society of Dynamic Games), 2001, vol. 6, pp. 253-273.

[50] S. Bhattacharya, T. Başar, and M. Falcone, "Numerical approximation for a visibility based pursuit-evasion game," in IEEE/RSJ Int. Conf. Intell. Robot. Syst., 2014, pp. 68-75.

[51] S. S. Kumkov, S. L. Ménec, and V. S. Patsko, "Level sets of the value function in differential games with two pursuers and one evader Interval analysis interpretation," Math. Comput. Sci., vol. 8, pp. 443-454, Aug. 2014.

[52] H. Zhang, L. Cui, and Y. Luo, "Near-optimal control for nonzero-sum differential games of continuous-time nonlinear systems using singlenetwork ADP,' IEEE Trans. Cybern., vol. 43, no. 1, pp. 206-216, Feb. 2013.

[53] D. Zhao, Q. Zhang, D. Wang, and Y. Zhu, "Experience replay for optimal control of nonzero-sum game systems with unknown dynamics," IEEE Trans. Cybern., 2015, accepted.

[54] I. M. Mitchell, A. M. Bayen, and C. J. Tomlin, "A time-dependent Hamilton-Jacobi formulation of reachable sets for continuous dynamic games," IEEE Trans. Autom. Control, vol. 50, no. 7, pp. 947-957, Jul. 2005.

[55] E. A. Cross and I. M. Mitchell, "Level set methods for computing reachable sets of systems with differential algebraic equation dynamics," in Proc. Amer. Control Conf., 2008, pp. 2260-2265.

[56] S. Mehraeen, T. Dierks, S. Jagannathan, and M. L. Crow, "Zero-sum two-player game theoretic formulation of affine nonlinear discrete-time systems using neural network," IEEE Trans. Cybern., vol. 43, no. 6, pp. $1641-1655$, Dec. 2013.

[57] V. Hekami and M. Dehghan, "Learning stationary correlated equilibria in constrained general-sum stochastic games," IEEE Trans. Cybern., 2015, accepted.

[58] Y. Hu, Y. Gao, and B. An, "Multiagent reinforcement learning with unshared value functions," IEEE Trans. Cybern., vol. 45, no. 4, pp. 647-662, Apr. 2015.

[59] Y. Hu, Y. Gao, and B. An, "Accelerating multiagent reinforcement learning by equilibrium transfer," IEEE Trans. Cybern., vol. 45, no. 7, pp. 1289-1302, Jul. 2015 .
[60] Z. Wu, J. Yu, Z. Su, M. Tan, and Z. Li "Towards an Esox lucius inspired multimodal robotic fish," Sci. China Inf. Sci., vol. 58, no. 5, pp. 1-13, May. 2015

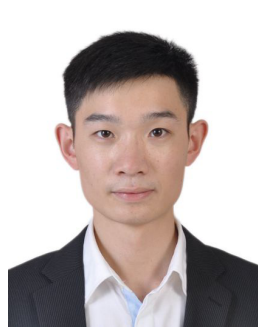

Wenzhong Zha (S'13) received the B.S. degree in mathematics from School of Science, Beijing Institute of Technology, Beijing, China, in 2008. $\mathrm{He}$ is currently a Ph.D. candidate at the School of Automation, Beijing Institute of Technology, Beijing, China.

His research interests include differential games, dynamic games, multi-agent systems, multiobjective optimization and decision, and incomplete information processing.

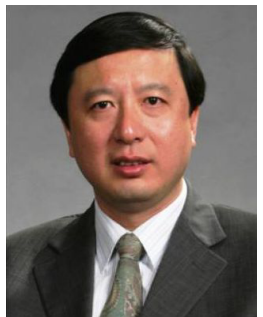

Jie Chen (M'09-SM'12) received the B.S. degree, the M.S. and Ph.D degrees from Beijing Institute of Technology, Beijing, China, in 1986, 1966, and 2001, respectively.

$\mathrm{He}$ is a Professor with the School of Automation, Beijing Institute of Technology, Beijing, China. His current research interests include complex systems, multi-agent systems, multi-objective optimization and decision, constrained nonlinear control, and optimization methods. Corresponding author of this paper.

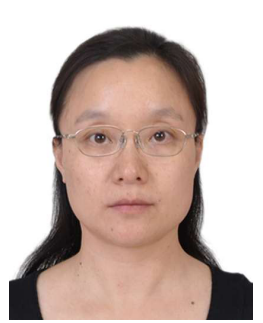

Zhihong Peng (M'12) received the B.S. degree from the Xiangtan Mining Institute (Now Hunan University of Science and Technology), Xiangtan, China, in 1995, and the Ph.D. degree from the Central South University, Changsha, China, in 2000.

She held one Post-Doctoral appointment at the Beijing Institute of Technology, Beijing, China. Since 2012, she has been a Professor with the Beijing Institute of Technology, Beijing, China. Her curren$\mathrm{t}$ research interests include intelligent information processing, multi-agent cooperation, optimization

and decision

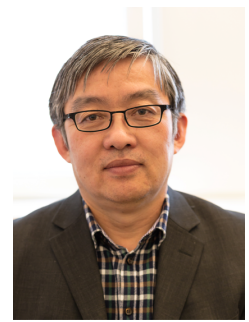

Dongbing Gu (SM'07) received the B.Sc. and M.Sc. degrees in control engineering from Beijing Institute of Technology, Beijing, China, and the Ph.D. degree in robotics from University of Essex, Essex, UK.

$\mathrm{He}$ was an Academic Visiting Scholar with the Department of Engineering Science, University of Oxford, Oxford, UK, from October 1996 to October 1997. In 2000, he joined the University of Essex as a Lecturer. Currently, he is a Professor with the School of Computer Science and Electronic Engineering, University of Essex. His current research interests include robotics, multi-agent systems, cooperative control, model predictive control, visual SLAM, wireless sensor networks, and machine learning. 